\title{
United Republic of Tanzania: Third Review Under the Policy Support Instrument and Request for Waivers and Modification of Assessment Criteria_Staff Report; Press Release on the Executive Board Discussion; and Statement by the Executive Director for the United Republic of Tanzania.
}

In the context of the third review under the policy support instrument and request for waivers and modification of assessment criteria, the following documents have been released and are included in this package:

- $\quad$ The staff report for the third review under the Policy Support Instrument and request for waivers and modification of assessment criteria, prepared by a staff team of the IMF, following discussions that ended on November 7, 2011, with the officials of Tanzania on economic developments and policies. Based on information available at the time of these discussions, the staff report was completed on December 23, 2011. The views expressed in the staff report are those of the staff team and do not necessarily reflect the views of the Executive Board of the IMF.

- $\quad$ A Press Release summarizing the views of the Executive Board as expressed during its January 20, 212 discussion of the staff report that completed the request and/or review.

- $\quad$ A statement by the Executive Director for the United Republic of Tanzania.

The documents listed below have been or will be separately released.

Letter of Intent sent to the IMF by the authorities of the United Republic of Tanzania*

Memorandum of Economic and Financial Policies by the authorities of the United Republic of Tanzania*

Technical Memorandum of Understanding*

*Also included in Staff Report

The policy of publication of staff reports and other documents allows for the deletion of market-sensitive information.

Copies of this report are available to the public from

International Monetary Fund • Publication Services

$70019^{\text {th }}$ Street, N.W. • Washington, D.C. 20431

Telephone: (202) 623-7430 • Telefax: (202) 623-7201

E-mail: publications@imf.org Internet: http://www.imf.org

\section{International Monetary Fund Washington, D.C.}


INTERNATIONAL MONETARY FUND

UNITED REPUBLIC OF TANZANIA

\title{
Third Review Under the Policy Support Instrument and Request for Waivers and Modification of Assessment Criteria
}

\author{
Prepared by the African Department \\ (In consultation with other departments)
}

Approved by Saul Lizondo and Dominique Desruelle

December 23, 2011

- $\quad$ Fund relations: A 3-year PSI-supported program was approved by the Executive Board on June 4, 2010. The first review was completed on December 3, 2010, and the second review on May 6, 2011.

- $\quad$ Staff team: Messrs. Allum (head), Lakwijk, Gaertner (all AFR), Papageorgiou (SPR), Ms. Mineshima (FAD), Mr. Wakeman-Linn (senior resident representative).

- Discussions: Held in Washington, DC during September 19-22 and in Dar es Salaam during October 26-November 7. The team met with Minister for Finance Mkulo; Governor Ndulu of the Bank of Tanzania; acting Permanent Secretary of the Treasury, Mr. Likwelile; other senior officials; and representatives of the private sector, civil society, development partners, and the press. Mr. Ndyeshobola (OED) participated in the discussions.

- $\quad$ Review: Staff supports the authorities' request for waivers of the nonobservance of end-June 2011 assessment criteria on net domestic financing and on net international reserves based on the corrective actions taken by the authorities. Staff also supports modification of assessment criteria for end-December 2011 for net international reserves and external debt, and recommends completion of the review. 


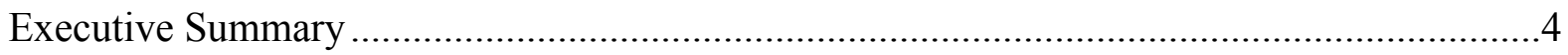

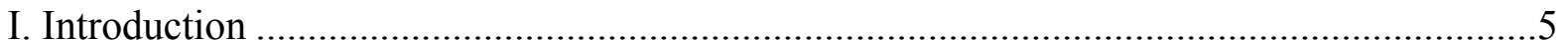

II. Recent Developments and Economic Outlook................................................................

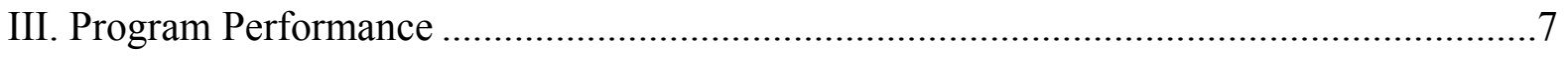

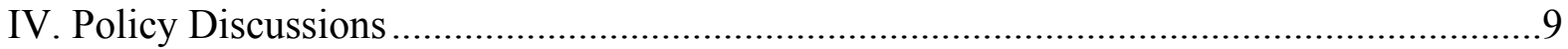

A. Reducing the Fiscal Deficit in 2011/12..............................................................

B. Creating Fiscal Space for Infrastructure Spending .................................................10

C. Fiscal and Other Structural Reforms ………………….....................................12

D. Monetary, Exchange Rate, and Financial Sector Policies ........................................14

V. Program Design and Monitoring................................................................................15

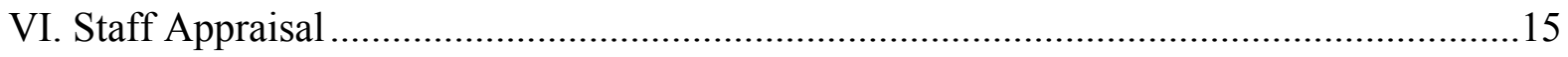

Tables

1. Selected Economic and Financial Indicators, 2009/09-2013/14 …..................................17

2a. Central Government Operations, 2009/09-2013/14 .......................................................18

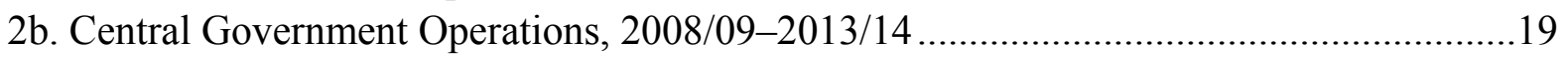

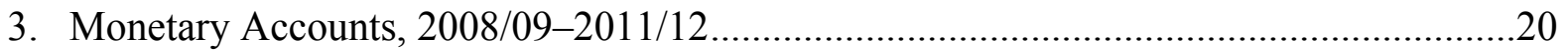

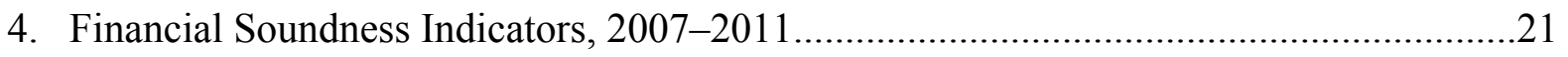

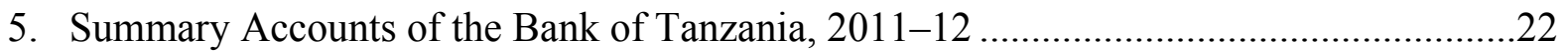

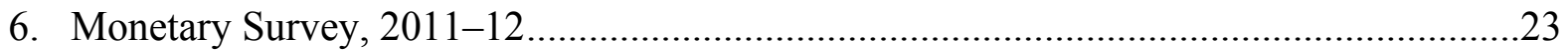

7. Balance of Payments, 2008/09-2013/14 ......................................................................24

8. Quantitative Assessment Criteria Under the Policy Support Instrument, June and

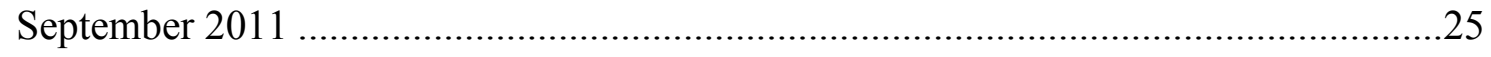

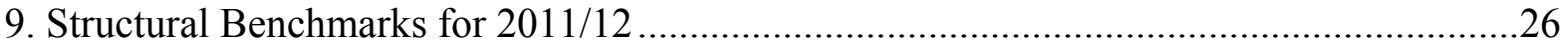

Figures

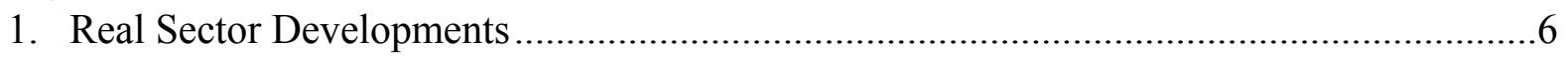

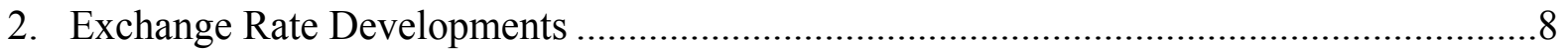

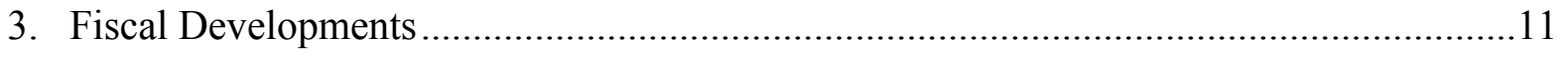

Box

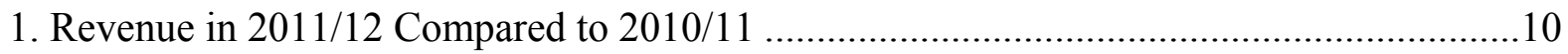


Appendix

Letter of Intent

Attachment

I. Memorandum of Economic and Financial Policies...............................................29

II. Technical Memorandum of Understanding on Selected Concepts and Definitions Used in the Monitoring of the PSI-Supported Program ......................................................44 


\section{EXECUTIVE SUMMARY}

- GDP growth is projected to remain strong at around 6 percent in 2011/12 (July-June), despite the impact of domestic power shortages and a softening world economy. Consumer price inflation rose during 2011, reaching 19 percent in November, largely due to external developments. Steady monetary management has helped keep core inflation in single digits, and headline inflation is projected to fall to about 9 percent by June 2012 .

- Program performance was mixed. Assessment criteria for June 2011 on domestic financing of the government and international reserves were missed, in part because of a slow start-up of planned external nonconcessional borrowing. Structural reforms continue, albeit with delays in some areas.

- Fiscal policy is being tightened for 2011/12 because of the deteriorating financing climate since mid-2011 and because of rising inflation. The authorities are eliminating non-priority recurrent spending and delaying some development spending to next year. This will reduce the projected overall fiscal deficit for the current fiscal year from a budgeted $7 \frac{1}{2}$ percent of GDP to $6 \frac{1}{2}$ percent of GDP. Further tightening of fiscal policies will be needed in the 2012/13 budget to further reduce the overall deficit and to keep net domestic financing at low levels. The focus should be on strengthening the revenue base and streamlining recurrent spending.

- Monetary conditions have been tightened. The Bank of Tanzania has tightened its reserve money program for 2011/12, and domestic interest rates have risen sharply since mid-2011. The exchange rate depreciated 12 percent against the dollar through October before stabilizing after interest rates rose. Financial soundness indicators are generally favorable, with a nonperforming loan ratio of 8 percent.

- The authorities are expanding Tanzania's electric power generation capacity and raising electricity tariffs. A review of the tariff structure is expected to result in an increase of at least 40 percent by end-2011 to help pay for emergency near-term generating costs and to strengthen power utility finances for the medium term.

- Tanzania has good prospects as a major producer of natural gas by the end of the decade. The authorities will identify the critical macroeconomic policies and institutions to successfully manage future gas wealth.

- The global outlook and domestic power sector represent key risks to the program. Although Tanzania weathered the global financial crisis well, it entered the global downturn in a stronger fiscal position than at present. A second, broader shock affecting investments in Africa's resource economies could pose risks to fiscal and debt sustainability and growth prospects. Steps to strengthen power sector finances will be important to limit risks to the budget. 


\section{INTRODUCTION}

1. Tanzania's second 3-year PSI has supported the authorities' economic program since mid-2010. The program's objectives include a progressive reduction of the fiscal deficit and reorienting spending from recurrent outlays to infrastructure investment, while preserving critical social expenditures. Structural reforms seek to enhance the returns to public spending and deepen the financial sector.

2. The economy has performed strongly over the last decade. Some progress has been made towards meeting the MDGs, in particular in the education and health sectors. Per capita income, however, is only about $\$ 550$, reflecting earlier decades of stagnation and high population growth. In the elections of October 2010, the President won a second term and his party retained parliamentary control, albeit with a smaller majority.

\section{RECENT DEVELOPMENTS AND ECONOMIC OUTLOOK}

\section{Macroeconomic trends remain generally favorable.}

- GDP growth eased from 71/4 percent in 2009/10 to 6 $1 \frac{1}{2}$ percent in 2010/11 (Table 1 and Figure 1). ${ }^{1}$ After low 2010 rainfalls cut hydroelectric generation, businesses largely weathered the 40 percent reduction in the national power supply by using back-up generators. Growth was high in transport and communication, construction, and financial intermediation. Traditional and manufacturing exports grew strongly and helped maintain balance in the external accounts.

- Consumer price inflation increased from 51/2 percent (year-on-year) at end-2010 to 19 percent in November 2011 largely due to external developments - substantially higher oil import prices than a year earlier and pressures on regional food supplies and prices resulting from drought in the Horn of Africa. Core inflation (which excludes food and energy prices) also rose, but remained in single digits at $83 / 4$ percent in November 2011.

\section{The fiscal deficit and recurrent spending in 2010/11 exceeded program}

projections (Table 2). The deficit was about 7 percent of GDP (cash basis), compared with a programmed $6 \frac{1}{2}$ percent of GDP. Revenue collections were slightly higher than programmed, with good performance in excise and income taxation. Recurrent spending, on the other hand, exceeded PSI projections by 1 percent of GDP, reflecting a lack of success in compressing goods, services and transfers spending as much as had been programmed. ${ }^{2}$ At the same time,

\footnotetext{
${ }^{1}$ The fiscal year begins July 1.

${ }^{2}$ Transfers are made to local governments which provide many education and health services.
} 
Figure 1. Real Sector Developments

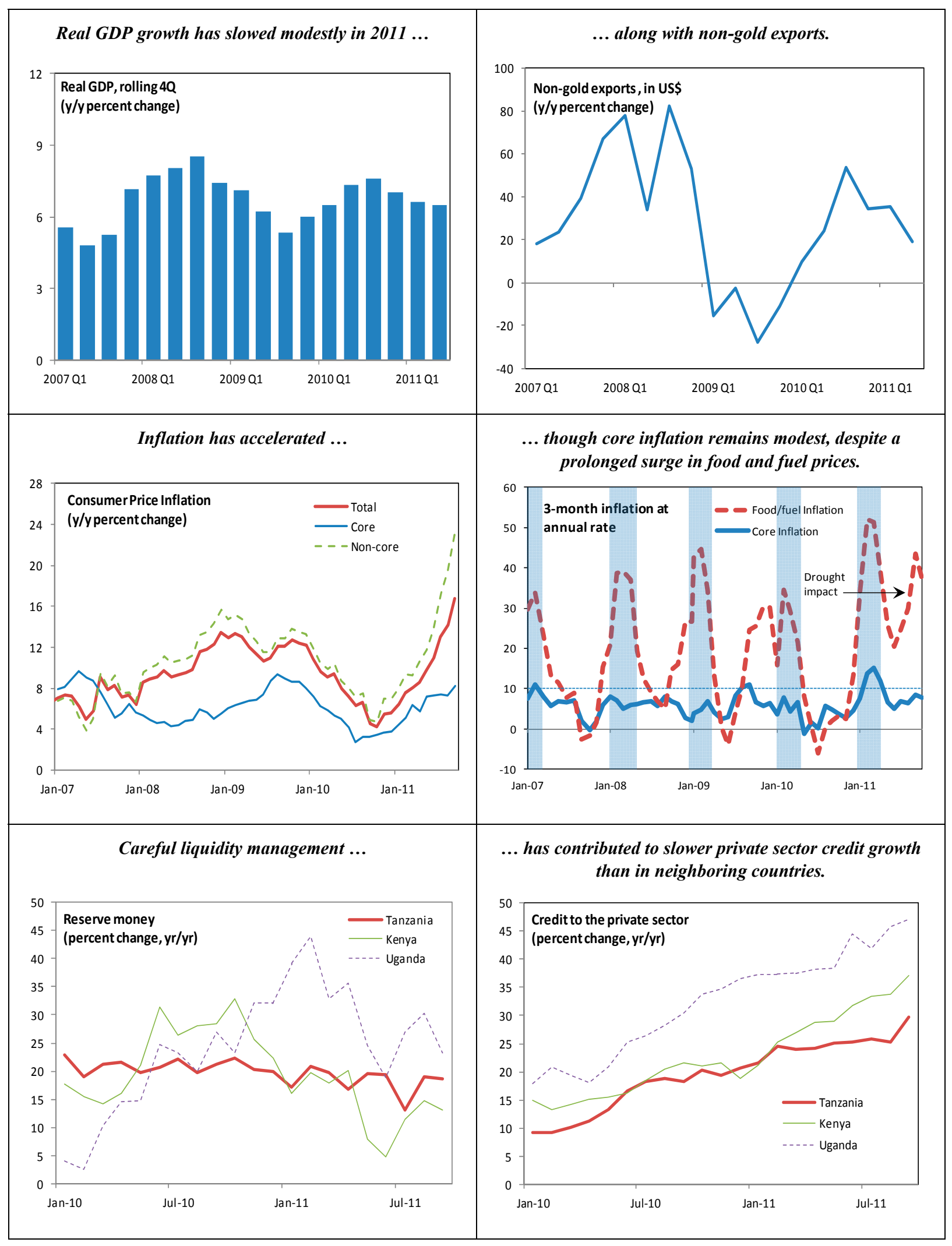


domestic payment arrears equivalent to about 1 percent of GDP were carried over into $2011 / 12$, largely in respect of road projects.

5. Monetary conditions have been tightened. In an environment of rising inflation and government financing needs, the Bank of Tanzania adhered closely to its monetary program. As a result, short-term interest rates rose sharply in recent months and the shilling, which depreciated during most of 2011, stabilized in November (Figure 2).

6. Growth in 2011/12 is projected to ease to 6 percent under the impact of domestic power shortages and a softening world economy. Efforts to fully restore power generation are underway but will take time to be implemented. For 2012/13, a gradual growth recovery is projected, assuming that energy issues are addressed and in the absence of negative external shocks. Inflation is projected to fall to 9 percent (year-on-year) by end-June 2012 as fuel prices level off, recent good harvests moderate food price increases, and the effects of tighter monetary and fiscal policies become visible. The international reserves cover, while continuing to decline gradually, is projected to remain close to 4 months of imports.

\section{Important downside risks relate to the global economy and domestic power} sector. Tanzania's economy weathered the global financial crisis well, reflecting its limited reliance on global trade and financing and domestic stimulus made possible by low inflation, sufficient fiscal and international reserves buffers, and additional official financing. The current scope for stimulus is, by contrast, limited, given high inflation and the increased size of fiscal deficits. The challenge now is to maintain tight liquidity conditions and gradually rebuild fiscal buffers to provide scope for flexibility in the event of further adverse external shocks. Power shortages are being addressed with new thermal capacity, and it will be important that tariffs reflect the new cost structure to avoid risks to public finances.

\section{Program Performance}

\section{8. $\quad$ PSI program performance was mixed.}

- $\quad$ Three out of five assessment criteria for end-June 2011 were met (Table 8). Reflecting a slower-than-expected start in tapping commercial credits, external nonconcessional borrowing was well below the PSI ceiling (by $12 / 3$ percent of GDP); no external arrears were accumulated; and reserve money was kept below its PSI ceiling.

- $\quad$ Two end-June 2011 assessment criteria were missed. The shortfall in external nonconcessional borrowing contributed both to a shortfall in international reserves ( $3 / 4$ percent of GDP) and higher-than-programmed domestic borrowing (2 $1 / 2$ percent of GDP). With strengthened treasury practices, domestic borrowing by the government also rose (by about 1 percent of GDP) to cover end-year payments of invoices that, with earlier procedures, would have carried over into the following fiscal year 
(MEFP q9). ${ }^{3}$ However, domestic borrowing by the government would have been 1 percent of GDP less if recurrent spending had been as programmed.

- $\quad$ All but one of the end-September 2011 indicative targets was met. Net international reserves fell short of programmed levels, reflecting a carryover from the June shortfall, delays in donor disbursements, and domestic arrears payments to foreign contractors (MEFP \10).

Figure 2. Exchange Rate Developments

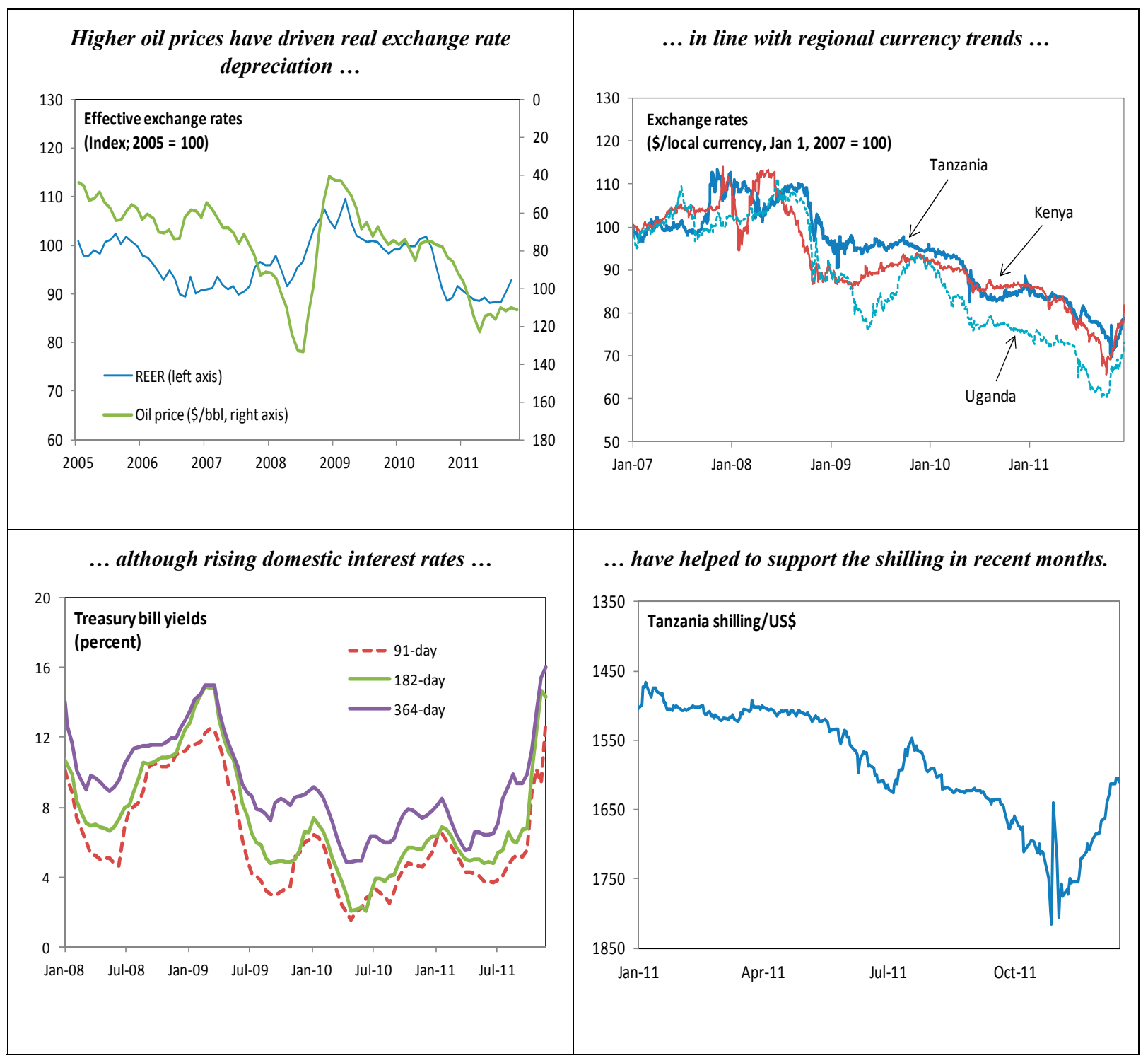

\footnotetext{
3 "MEFP" refers to the Memorandum of Economic and Financial Policies of December 2011 attached to this report.
} 
9. Structural reforms continued, albeit with some delay. Three out of five benchmarks for 2011 have been met (Table 9). The authorities propose to delay until March 2012 the issuance of investment guidelines for pension funds; these are to be based on the findings of an actuarial review of existing social security funds (which will assess the extent of any financial problems in the funds) that has been delayed by a slow build up of capacity at the newly-established Social Security Regulatory Authority. The authorities have also requested that the benchmark on approval of a new social protection framework be dropped; this is now being tackled as part of a broader reform of social protection (MEFP -11). In the context of the next PSI review, staff will discuss with the authorities the possible establishment of an indicative target on social spending.

\section{Policy Discussions}

\section{A. Reducing the Fiscal Deficit in 2011/12}

\section{The approved 2011/12 budget was more expansionary than envisaged in the} second PSI review. ${ }^{4}$ The authorities noted that Tanzania has significant spending needs on education, health, and road infrastructures. Teacher recruitment needs to keep pace with recent success in raising school enrollment; advances in the health sector, including construction of new clinics, require higher levels of recurrent spending; and Tanzania is undertaking an ambitious program to improve its infrastructures, particularly roads. ${ }^{5}$ As a result, the 2011/12 budget envisaged a fiscal deficit of 7.6 percent of GDP, up from 6.0 percent of GDP in the second PSI review. The authorities calculated that this deficit could be financed within the PSI limit on net domestic financing of the government by carrying over to 2011/12 the portion of programmed external nonconcessional borrowing that was not used in 2010/11.

11. With downside risks to external financing and inflationary pressures to address, the government is reining in the 2011/12 budget. The overall deficit is being reduced to a projected 6.6 percent of GDP, largely through expenditure savings. Non-priority recurrent spending will be reduced by one-half percent of GDP, while keeping social spending unchanged (MEFP 『17). A similar amount of domestically financed development spending will be delayed to $2012 / 13$, focusing on projects where delays do not trigger contractor penalties or undermine project effectiveness. Revenues are projected to increase by $1 \frac{1}{4}$ percentage points of GDP from 2010/11 levels, reflecting improvements in tax administration, measures adopted in the 2011/12 budget, and other factors (Box 1).

\footnotetext{
${ }^{4}$ Country Report No. 11/105.

${ }^{5}$ The 2011/12 budget includes funds for the elimination of arrears (largely in road building) amounting to 1 percent of GDP that were carried over from the previous year (MEFP $\mid 26)$.
} 


\section{Box 1. Revenue in 2011/12 compared to 2010/11}

- Stronger tax administration. Increased use is being made of electronic fiscal devices to reduce VAT fraud (projected yield of 0.3 percent of GDP) and efforts have been stepped up to resolve mining tax disputes and improve mining tax administration ( 0.2 percent of GDP).

- Tax measures. The 2011/12 budget eliminated some VAT exemptions, raised selected excise tax rates, and extended excises to bottled water (yield of 0.3 percent of GDP).

- Nontax revenue ( 0.9 percent of GDP). Bank of Tanzania dividend payments to the budget, volatile but typically small, will be 0.4 percent of GDP in 2011/12 (reflecting revaluationbased capital gains in 2010/11), and higher business license fees add 0.5 percent of GDP. A smaller dividend payment is projected to reduce nontax revenue in 20112/13.

- Offsets (negative 0.4 percent of GDP). Power shortages are projected to negatively affect income tax collections, while excise tax revenue (high in 2010/11) may return to more normal levels.

12. The 2011/12 deficit will be financed by domestic borrowing of 1 percent of GDP (as previously programmed) and US\$575 million of external borrowing. The latter figure is slightly higher than previously programmed, but programmed external nonconcessional borrowing for the combined two-year budget period (US\$675 million) is well below the originally-envisaged US\$1.05 billion. Through October 2011, the authorities contracted nonconcessional debts of about US\$450 million, primarily for infrastructure projects in the power and road sectors. The program ceiling on external nonconcessional borrowing through end-June 2012 (US\$1.05 billion) provides for the above budget financing, as well as potential public enterprise borrowing guaranteed by the government, such as for the gas pipeline discussed below. ${ }^{6}$

\section{B. Creating Fiscal Space for Infrastructure Spending}

\section{A further gradual reduction in the overall fiscal deficit to about $5 \frac{1}{2}$ percent of} GDP is projected for 2012/13. Net domestic financing would be maintained at 1 percent of GDP while external nonconcessional borrowing to strengthen public infrastructures would be within the originally programmed envelope. To achieve this deficit goal, current policies would need to be strengthened by about 1 percent of GDP (Table 2), through further revenue raising and/or expenditure trimming measures. ${ }^{7}$

\section{A key focus for the 2012/13 and subsequent budgets will be on the level and} affordability of recurrent spending (Figure 3). Recurrent spending has expanded by more

\footnotetext{
${ }^{6}$ The program would accommodate commercial borrowing for budget purposes in excess of $\$ 575$ million for $2011 / 12$, provided that this is offset by a lower level of net domestic financing.

${ }^{7}$ This required fixed adjustment is reported as "unidentified fiscal measures" in Tables $2 \mathrm{a}$ and $2 \mathrm{~b}$.
} 
Figure 3. Fiscal Developments

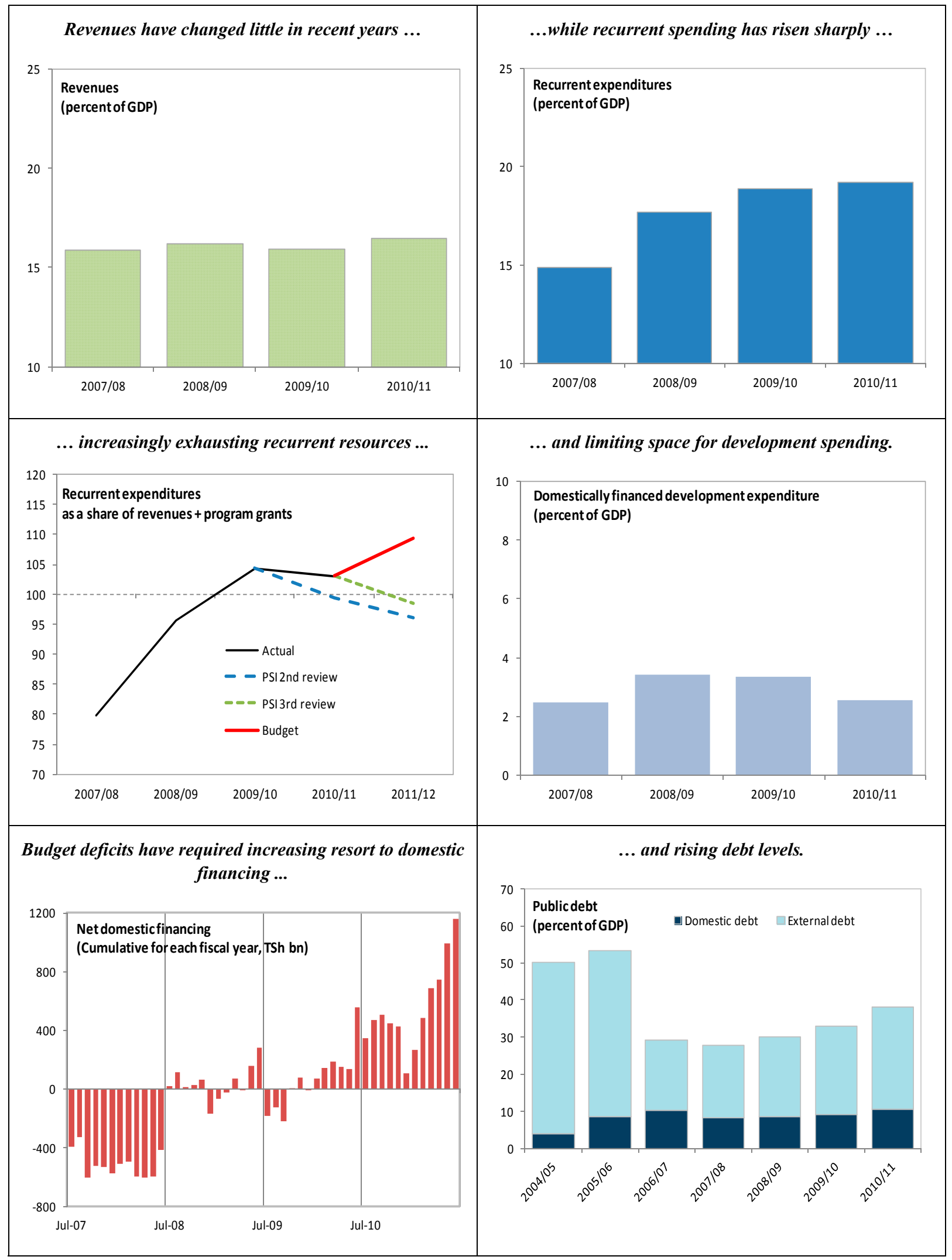


than 4 percentage points of GDP over the last four years. Central government wages and allowances have risen strongly, while the program to decentralize social spending has become increasingly costly. With only limited growth in government revenues, recurrent spending has recently been partly funded by borrowing, crowding out financing for development spending. For the 2012/13 and 2013/14 budgets, the authorities intend to put recurrent spending onto a more sustainable footing by slowing its growth relative to revenue (MEFP \19). To this end, they identified areas for possible expenditure savings and revenue gains (MEFP q21-22).

\section{Fiscal and Other Structural Reforms}

15. The authorities continue to improve their public expenditure management. They explained that their cash budgeting system, which recently moved from a one-month to a three-month planning horizon to more smoothly implement recurrent expenditures, has been effective in enforcing spending ceilings. They were therefore confident that the plans to curtail non-priority recurrent spending would be achieved. The authorities indicated that they regarded the amount of expenditure arrears carried over into 2011/12 (around 1 percent of GDP) as exceptional, related to a temporary surge in road program commitments in $2010 .^{8}$ They recognized the importance of realistic budgeting, and indicated that the significantly expanded 2011/12 budget for the roads sector and the absence of new investment projects this year would be sufficient to settle past arrears and meet obligations on existing projects, without carrying new arrears into 2012/13. As of early December 2011, 85 percent of the 2010/11 road sector arrears had been settled out of the 2011/12 budget allocation, and the remainder will be cleared in early 2012. A new phase of public financial management reform will strengthen audits and enhance budget control and oversight (MEFP q23-25). The authorities are committed to publishing the complete budget documents on the Ministry of Finance website to enhance budget transparency (structural benchmark, MEFP $\mid 28$ ).

16. On debt management, a unified single debt database was launched in 2010/11 in the Ministry of Finance to maintain quality debt data under one roof. To help bolster Tanzania's debt management capacity, the authorities have requested additional technical assistance on their Medium-Term Debt Strategy, the first version of which has been published.

\section{The authorities are expanding Tanzania's electric power generation capacity.} The shortfall in hydroelectric power generation is being tackled by installing new thermal capacity, some on a temporary basis. The generating cost for this new capacity is high, but overall costs will come down when hydro capacity recovers and as additional low cost natural gas powered generation comes on line.

\footnotetext{
${ }^{8}$ Arrears data collection was strengthened in $2010 / 11$ as arrears were recognized as a potential problem. Data on arrears carried over into 2010/11 from the previous year are not available.
} 
18. Power tariffs are being increased. The state power utility, TANESCO, has calculated that power tariffs would need to more than double to cover the present cost structure. However, as costs are projected to come down in the year ahead, the government plans to adopt a tariff increase of no less than 40 percent by end-2011 (structural benchmark, MEFP Table 4), which would cover part of the higher near-term generating costs while strengthening TANESCO finances for the medium-term. ${ }^{9}$ Temporary operating losses will be financed by medium-term domestic bank borrowing by TANESCO of no more than 1 percent of GDP, guaranteed by the government. Updated projections of energy generating costs, including based on rainfall in the coming season and its impact on hydroelectric capacity, will be a focus of the next PSI review. The government is committed to a tariff structure that ensures TANESCO’s financial viability (MEFP \$33).

19. Building a gas pipeline for $\mathbf{\$ 1 . 2}$ billion is being considered. Financing for the project (equivalent to $4 \frac{1}{2}$ percent of GDP) would likely be contracted in 2012/13 from Chinese banks. The financing - comprising a 70-30 combination of concessional and nonconcessional loans - would not pose risks to debt sustainability, being structured to comply with the PSI nonconcessional borrowing limits. ${ }^{10}$ The project, to be implemented largely within 2012/13, would transport gas from domestic fields to electric power plants. The authorities view this project as high value added and will share its technical and economic evaluation with staff (structural benchmark, MEFP q34). ${ }^{11}$

\section{Tanzania has good prospects as a major producer of natural gas in about a}

decade. This could result in multi-billion dollar FDI over the next 5 years and the start of correspondingly large export and budget revenue flows around 2020. The authorities intend

\footnotetext{
${ }^{9}$ Given the low share of electricity in household consumption (only 15 percent of the population has access to electricity), the direct impact on the Consumer Price Index will be limited. Second-round effects through business operating costs will also be modest as the improved power supply from thermal generators will reduce the need for businesses to operate costly back-up generators.

${ }^{10}$ The project would add around 2 percent of GDP in debt relative to the last DSA and significant margins would remain under DSA thresholds.

${ }^{11}$ A planned coal/iron ore investment in Tanzania of up to $\$ 3$ billion (11 percent of 2012/13 GDP) by a Chinese company is still in the preparatory stages. The project would mine iron ore and smelt it with electricity produced from local coal; surplus power would be sold to the Tanzanian market. The project would not involve external commercial borrowing or guarantees by the government.
} 
to review over the coming year their macroeconomic policies and institutions to successfully manage future gas wealth (structural benchmark, MEFP q35).

\section{Monetary, Exchange Rate, and Financial Sector Policies}

21. The Bank of Tanzania has taken steps to tighten monetary conditions. The rise in inflation during 2011 was initially driven by supply-side factors, but in recent months an adverse inflation and exchange rate dynamic threatened to take hold. In response, the Bank of Tanzania lowered its 2011/12 target for reserve money growth from 19 to 18 percent with a view to keeping core inflation in single digits. In addition, the minimum reserve requirement on government deposits in the banking system was increased from 20 to 30 percent. The Bank of Tanzania will continue to manage liquidity under its reserve money targeting framework through a combination of open market operations and regular foreign exchange sales, while allowing the exchange rate to remain market determined (MEFP q38). To dampen activity that might have added to the downward pressure on the currency, in October the Bank of Tanzania reduced the limit on banks' net open foreign exchange positions from 20 to 10 percent of core capital, and tightened enforcement of existing restrictions on lending to nonresidents. The monetary and exchange rate measures, combined with disciplined implementation of the reserves money ceiling, was associated with a rise in overnight interbank interest rates to the 30 percent range in December, reversing the earlier currency weakness (text figure).

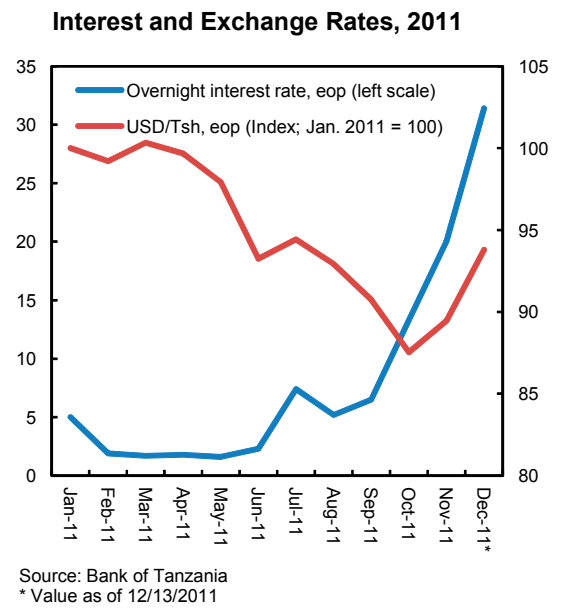

22. Financial soundness indicators remain broadly favorable. Nonperforming loans were 8 percent of total loans at end-September, down from nearly 10 percent at the beginning of the year reflecting efforts to strengthen loan collection and follow-up and tighten underwriting standards. The sector remains well-capitalized, with overall capital of 17.4 percent of risk-weighted assets. The Bank of Tanzania has also stepped up enforcement to ensure compliance with regulations on loan classification and loss recognition, particularly for banks with higher levels of nonperforming loans, and revised its risk-based supervisory framework to reflect international best practice.

\section{Financial sector reforms have continued to move forward, in line with the} recommendations from the 2009 FSAP. A financial regulators forum has been launched to coordinate crisis management, and the Bank of Tanzania has made further improvements in its framework for financial stability monitoring. Work has also begun on the creation of a credit databank and national identification registry, which will provide the basis for establishing private credit reference bureaus; at present, the inability of banks to monitor the credit history of potential borrowers remains a major obstacle in expanding financial access. 
In addition, the Social Security Regulatory Authority, which became operational in 2010, is close to the completion of an actuarial review of existing social security funds that will provide the basis for investment guidelines for the sector to be issued in early 2012 (delayed structural benchmark, MEFP $\lceil 40)$.

24. Legislation will be submitted to parliament to tighten the anti-money laundering framework (structural benchmark, MEFP -46). This would respond to the citation of Tanzania by the international Financial Action Task Force in October 2011 for having made insufficient progress in improving policies on anti-money laundering and countering the financing of terrorism.

\section{Program Design ANd Monitoring}

25. Quantitative assessment criteria for end-December 2011 and end-June 2012 are presented in MEFP Table 2. Structural benchmarks are included in MEFP Tables 3 and 4. The assessment criterion on net international reserves is changed from measuring on a stock basis to a flow basis to eliminate base effects. The assessment criterion on external debt is changed to make it cumulative from the beginning of the program instead of the fiscal year to allow for more flexibility in the timing of contracts within the agreed 3-year program ceiling.

\section{Staff Appraisal}

26. Tanzania weathered the global financial crisis well, in part due to countercyclical fiscal policies. However, with higher inflation and fiscal deficits, there is now limited scope for expansionary policies to counter a renewed external shock. Accordingly, the priority is to gradually start rebuilding fiscal buffers. Staff welcomes the authorities' decision to tighten the 2011/12 fiscal stance. Strict expenditure control will be important to avoid carrying over arrears to $2012 / 13$.

27. Further policy adjustment will be needed in 2012/13 to further reduce the fiscal deficit and strengthen debt sustainability. To create fiscal space for infrastructure investment, it will be important to boost Tanzania's modest revenue base while also streamlining recurrent spending programs. The latter will require better prioritization across programs, as well as improved efficiency and value for money in public spending, not only within the central government, but also in the local governments.

28. The power sector should not become a drain on the budget. Borrowing by the state power utility to finance a projected temporary spike in power generating costs should be regarded as exceptional and kept to limited amounts. Following the planned end-2011 increase in power tariffs, the need for further adjustments should be kept under close review to safeguard the power utility's finances and ensure its ability to repay new borrowing.

29. The authorities should stand ready to further tighten liquidity if inflationary

pressures build. The core rate of inflation and risks from the exchange rate should be 
monitored closely. If needed, policy tightening should be prompt to avoid a build up of currency market pressure. Staff encourages the authorities to maintain a level of international reserves that would continue to provide a buffer against external shocks. The floating exchange rate has served Tanzania well.

30. Policy implementation should be alert to risks from the global economy. Although Tanzania is benefiting from strong terms of trade and growing resource-related investments, a renewed downturn in the global economy would require efforts to trim spending ambitions in anticipation of weaker revenue growth and more limited external financing options.

31. Staff recommends the completion of the third review under the PSI. The authorities' policy commitments provide confidence that the program remains on track and slippages will not recur. Staff supports the authorities' request for waivers for the missed assessment criteria on net international reserves and net domestic financing based on steps taken to rein in the fiscal deficit through cuts in non-priority recurrent spending, while also developing a more reliable external borrowing pipeline. Staff also supports the proposed rephasing of a structural benchmark and elimination of another, modification of assessment criteria for end-December 2011 for net international reserves and for external debt, and setting of end-June 2012 assessment criteria. 
Table 1. Tanzania: Selected Economic and Financial Indicators, 2008/09-2013/14

\begin{tabular}{|c|c|c|c|c|c|c|c|c|}
\hline \multirow[t]{2}{*}{. } & \multirow[t]{2}{*}{$2008 / 09$} & \multirow[t]{2}{*}{$2009 / 10$} & \multicolumn{2}{|c|}{$\underline{2010 / 11}$} & \multicolumn{2}{|c|}{$\underline{2011 / 12}$} & \multirow{2}{*}{$\begin{array}{r}\text { 2012/13 } \\
\text { Proj. }\end{array}$} & \multirow{2}{*}{$\begin{array}{r}2013 / 14 \\
\text { Proj. }\end{array}$} \\
\hline & & & Prog. $^{5}$ & Prel. & Prog. $^{5}$ & Proj. & & \\
\hline & \multicolumn{8}{|c|}{ (Annual percentage change, unless otherwise indicated) } \\
\hline \multicolumn{9}{|l|}{ National income and prices } \\
\hline Real GDP growth & 6.2 & 7.3 & 6.5 & 6.5 & 6.6 & 6.0 & 7.2 & 7.5 \\
\hline Consumer prices (period average) & 11.8 & 10.5 & 6.3 & 7.0 & 7.0 & 13.7 & 5.8 & 5.0 \\
\hline Consumer prices (end of period) & 10.7 & 7.2 & 7.5 & 10.9 & 5.5 & 9.0 & 5.0 & 5.1 \\
\hline \multicolumn{9}{|l|}{ External sector } \\
\hline Export, f.o.b (in millions of U.S. dollars) & 3,268 & 3,799 & 4,683 & 4,888 & 5,188 & 5,865 & 6,648 & 7,499 \\
\hline Imports, f.o.b. (in millions of U.S. dollars) & $-6,220$ & $-6,570$ & $-7,873$ & $-8,012$ & $-8,750$ & $-9,039$ & $-10,034$ & $-10,931$ \\
\hline Export volume & 8.0 & 6.2 & 9.9 & 10.2 & 6.9 & 8.6 & 10.2 & 12.5 \\
\hline Import volume & 10.4 & 4.7 & 7.3 & 7.8 & 5.4 & 8.5 & 12.2 & 9.1 \\
\hline Terms of trade & 7.6 & 8.6 & 0.3 & 1.7 & -1.8 & 6.3 & 3.9 & 0.4 \\
\hline Nominal effective exchange rate (end of period; depreciation= -) & -3.7 & -3.7 & $\ldots$ & -17.7 & $\ldots$ & $\ldots$ & $\ldots$ & $\ldots$ \\
\hline Real effective exchange rate (end of period; depreciation= -) & 6.3 & 0.3 & $\ldots$ & -13.4 & $\ldots$ & $\ldots$ & $\ldots$ & $\ldots$ \\
\hline \multicolumn{9}{|l|}{ Money and credit } \\
\hline Broad money (M3) & 18.5 & 25.1 & 20.1 & 22.0 & 19.0 & 20.0 & 19.6 & 18.5 \\
\hline Net foreign assets & 15.8 & 25.3 & 19.1 & 10.2 & 10.1 & 15.9 & 7.3 & 7.3 \\
\hline Net domestic assets & 21.7 & 25.0 & 21.2 & 35.7 & 29.1 & 23.9 & 30.4 & 26.6 \\
\hline Credit to nongovernment sector & 33.1 & 16.7 & 23.0 & 25.2 & 20.8 & 23.0 & 24.0 & 22.1 \\
\hline Velocity of money (GDP/M3; average) & 3.6 & 3.4 & 3.2 & 3.2 & 3.1 & 3.0 & 2.8 & 2.7 \\
\hline \multirow[t]{2}{*}{ Treasury bill interest rate (in percent; end of period) ${ }^{1}$} & 7.0 & 3.3 & $\ldots$ & 4.8 & $\ldots$ & $\ldots$ & $\ldots$ & $\ldots$ \\
\hline & \multicolumn{8}{|c|}{ (Percent of GDP) } \\
\hline \multicolumn{9}{|l|}{ Public Finance } \\
\hline Revenue (excluding grants) & 16.2 & 15.9 & 16.1 & 16.5 & 16.3 & 17.8 & 17.5 & 17.7 \\
\hline Total grants & 5.1 & 4.6 & 6.4 & 4.7 & 6.4 & 6.6 & 5.1 & 4.8 \\
\hline Expenditure & 26.1 & 27.5 & 29.0 & 27.2 & 28.6 & 31.1 & 29.3 & 28.8 \\
\hline Overall balance (excluding grants) ${ }^{2}$ & -9.6 & -11.1 & -12.9 & -11.6 & -12.4 & -13.2 & -10.7 & -9.3 \\
\hline Overall balance (including grants) ${ }^{2}$ & -4.6 & -6.4 & -6.5 & -6.9 & -6.0 & -6.6 & -5.5 & -4.5 \\
\hline Domestic financing & 0.8 & 1.9 & 1.0 & 3.6 & 1.0 & 1.0 & 1.0 & 1.0 \\
\hline Domestic debt stock (end of period) ${ }^{3}$ & 8.4 & 9.1 & $\ldots$ & 10.7 & $\ldots$ & 10.3 & 10.2 & 10.0 \\
\hline \multicolumn{9}{|l|}{ Savings and investment } \\
\hline Resource gap & -12.7 & -11.8 & -12.6 & -11.8 & -12.4 & -11.7 & -11.1 & -10.4 \\
\hline Investment & 29.4 & 28.9 & 28.8 & 28.7 & 29.0 & 28.6 & 28.9 & 29.0 \\
\hline Government & 8.4 & 8.4 & 11.3 & 9.1 & 11.3 & 10.3 & 10.1 & 9.3 \\
\hline Nongovernment ${ }^{4}$ & 21.0 & 20.5 & 17.5 & 19.5 & 17.7 & 18.3 & 18.7 & 19.7 \\
\hline Gross domestic savings & 16.6 & 17.1 & 16.2 & 16.8 & 16.6 & 16.9 & 17.8 & 18.6 \\
\hline \multicolumn{9}{|l|}{ External sector } \\
\hline Current account balance (excluding current transfers) & -14.0 & -12.0 & -12.6 & -12.9 & -13.1 & -12.9 & -12.4 & -11.4 \\
\hline \multirow{2}{*}{ Current account balance (including current transfers) } & -10.7 & -9.2 & -9.5 & -9.8 & -10.7 & -10.0 & -9.7 & -8.9 \\
\hline & \multicolumn{8}{|c|}{ (Millions of U.S. dollars, unless otherwise indicated) } \\
\hline \multicolumn{9}{|l|}{ Balance of payments } \\
\hline Current account balance (excluding current transfers; deficit= -) & $-2,940$ & $-2,709$ & $-2,944$ & $-3,020$ & $-3,326$ & $-3,090$ & $-3,263$ & $-3,296$ \\
\hline Gross official reserves (end of period) & 2,930 & 3,483 & 3,831 & 3,610 & 4,133 & 3,868 & 4,076 & 4,260 \\
\hline In months of imports of goods and services (current year) & 4.5 & 5.0 & 4.7 & 4.4 & 4.5 & 4.1 & 4.0 & 3.8 \\
\hline External debt stock (end of period; percent of GDP) & 27.1 & 30.0 & 35.1 & 33.0 & 38.4 & 38.1 & 39.4 & 39.4 \\
\hline
\end{tabular}

Sources: Tanzanian authorities and IMF staff estimates and projections.

${ }^{1}$ End-year (June) monthly weighted-average yield of 35-, 91-, 182-, and 364-day treasury bills.

${ }^{2}$ Including adjustment to cash basis.

${ }_{4}^{3}$ Net of Treasury bills issued for liquidity management.

${ }^{4}$ Including change in stocks.

${ }^{5}$ From the second review under the PSI. 
Table 2a. Tanzania: Central Government Operations, 2008/09-2013/14 ${ }^{1}$

(Billions of Tanzania Shillings)

\begin{tabular}{|c|c|c|c|c|c|c|c|c|c|}
\hline & \multirow[t]{2}{*}{$2008 / 09$} & \multirow[t]{2}{*}{$2009 / 10$} & \multicolumn{2}{|c|}{$2010 / 11$} & \multicolumn{3}{|c|}{$2011 / 12$} & \multirow{2}{*}{$\frac{2012 / 13}{\text { Proj. }}$} & \multirow{2}{*}{$\begin{array}{r}2013 / 14 \\
\text { Proj. }\end{array}$} \\
\hline & & & Prog. ${ }^{6}$ & Actual & Prog. ${ }^{6}$ & Budget & Proj. & & \\
\hline Total revenue & 4,293 & 4,800 & 5,584 & 5,739 & 6,397 & 6,944 & 7,068 & 7,865 & 8,997 \\
\hline Tax revenue & 4,044 & 4,428 & 5,154 & 5,296 & 5,904 & 6,229 & 6,209 & 7,053 & 8,028 \\
\hline Import duties & 359 & 367 & 457 & 449 & 524 & 564 & 544 & 618 & 704 \\
\hline Value-added tax & 1,231 & 1,390 & 1,613 & 1,531 & 1,847 & 2,043 & 1,946 & 2,220 & 2,527 \\
\hline Excises & 762 & 838 & 885 & 1,052 & 1,014 & 1,105 & 1,080 & 1,227 & 1,396 \\
\hline Income taxes & 1,229 & 1,334 & 1,623 & 1,660 & 1,859 & 1,864 & 1,950 & 2,214 & 2,521 \\
\hline Other taxes & 463 & 499 & 576 & 604 & 660 & 654 & 689 & 773 & 880 \\
\hline Nontax revenue ${ }^{2}$ & 249 & 372 & 430 & 443 & 492 & 716 & 858 & 812 & 969 \\
\hline LGA & & 138 & 140 & 118 & 147 & 350 & 350 & 412 & 484 \\
\hline Other & 249 & 234 & 290 & 325 & 345 & 365 & 508 & 400 & 485 \\
\hline Total expenditure & 6,907 & 8,312 & 10,043 & 9,439 & 11,260 & 12,640 & 12,325 & 13,144 & 14,586 \\
\hline Recurrent expenditure & 4,681 & 5,700 & 6,269 & 6,690 & 6,690 & 7,714 & 7,717 & 8,715 & 9,856 \\
\hline Wages and salaries & 1,609 & 1,723 & 2,363 & 2,346 & 2,481 & 2,835 & 2,892 & 3,268 & 3,696 \\
\hline Interest payments & 243 & 249 & 287 & 353 & 469 & 309 & 456 & 512 & 578 \\
\hline Domestic & 208 & 208 & 211 & 285 & 343 & 221 & 334 & 328 & 364 \\
\hline Foreign $^{3}$ & 35 & 41 & 76 & 68 & 126 & 88 & 123 & 183 & 214 \\
\hline Goods and services and transfers ${ }^{2}$ & 2,830 & 3,728 & 3,619 & 3,991 & 3,740 & 4,570 & 4,368 & 4,936 & 5,583 \\
\hline Development expenditure & 2,226 & 2,611 & 3,774 & 2,749 & 4,570 & 4,926 & 4,609 & 4,429 & 4,730 \\
\hline Domestically financed & 906 & 1,005 & 1,366 & 985 & 1,573 & 1,871 & 1,714 & 1,749 & 1,826 \\
\hline Foreign (concessionally) financed & 1,320 & 1,607 & 2,408 & 1,764 & 2,997 & 3,054 & 2,894 & 2,680 & 2,905 \\
\hline Overall balance before grants & $-2,614$ & $-3,512$ & $-4,460$ & $-3,701$ & $-4,864$ & $-5,695$ & $-5,258$ & $-5,280$ & $-5,590$ \\
\hline Grants & 1,340 & 1,405 & 2,219 & 1,627 & 2,499 & 2,718 & 2,627 & 2,306 & 2,439 \\
\hline Program (including basket grants) ${ }^{4}$ & 798 & 924 & 1,089 & 1,062 & 931 & 1,093 & 1,211 & 1,202 & 1,263 \\
\hline Of which: basket grants & 194 & 258 & 345 & 335 & 392 & 392 & 444 & 461 & 485 \\
\hline Project & 462 & 439 & 861 & 370 & 1,338 & 1,149 & 908 & 952 & 1,000 \\
\hline MDRI (IMF) grant relief & 68 & 22 & 0 & 0 & 0 & 0 & 0 & 0 & 0 \\
\hline MCA funding & 12 & 20 & 269 & 196 & 229 & 476 & 507 & 152 & 176 \\
\hline Overall balance after grants & $-1,275$ & $-2,107$ & $-2,241$ & $-2,073$ & $-2,365$ & $-2,978$ & $-2,631$ & $-2,974$ & $-3,151$ \\
\hline Adjustment to cash ${ }^{5}$ & 60 & 167 & 0 & -320 & 0 & 0 & 0 & 0 & 0 \\
\hline Overall balance (cash basis) & $-1,215$ & $-1,940$ & $-2,241$ & $-2,393$ & $-2,365$ & $-2,978$ & $-2,631$ & $-2,974$ & $-3,151$ \\
\hline Unidentified fiscal measures & 0 & 0 & 0 & 0 & 0 & 0 & 0 & -487 & -884 \\
\hline Financing & 1,215 & 1,940 & 2,241 & 2,393 & 2,365 & 2,978 & 2,631 & 2,487 & 2,267 \\
\hline Foreign (net) & 956 & 1,380 & 1,864 & 1,149 & 1,972 & 2,410 & 2,234 & 2,038 & 1,760 \\
\hline Foreign loans & 984 & 1,448 & 1,907 & 1,192 & 2,099 & 2,477 & 2,351 & 2,270 & 2,158 \\
\hline Program (including basket loans) ${ }^{4}$ & 495 & 752 & 479 & 394 & 545 & 465 & 619 & 624 & 655 \\
\hline Of which: basket loans & 163 & 194 & 242 & 221 & 296 & 296 & 258 & 237 & 249 \\
\hline Project & 489 & 696 & 692 & 643 & 741 & 741 & 778 & 879 & 996 \\
\hline Nonconcessional borrowing & 0 & 0 & 736 & 154 & 812 & 1,272 & 954 & 767 & 507 \\
\hline Amortization & -27 & -68 & -43 & -43 & -127 & -67 & -117 & -232 & -397 \\
\hline Domestic (net) & 214 & 560 & 347 & 1,244 & 393 & 393 & 397 & 448 & 507 \\
\hline Bank financing & 214 & 585 & 297 & 907 & 353 & 393 & 357 & 398 & 457 \\
\hline Nonbank financing & 0 & -25 & 50 & 337 & 40 & 0 & 40 & 50 & 50 \\
\hline One-off transfers from agencies & 0 & 0 & 0 & 0 & 0 & 174 & 0 & 0 & 0 \\
\hline Privatization proceeds & 45 & 10 & 30 & 0 & 0 & 0 & 0 & 0 & 0 \\
\hline Amortization of parastatal debt & 0 & -9 & 0 & 0 & 0 & 0 & 0 & 0 & 0 \\
\hline \multicolumn{10}{|l|}{ Memorandum items: } \\
\hline Total public debt (in percent of GDP) ${ }^{7}$ & 29.9 & 32.8 & $\ldots$ & 37.5 & $\ldots$ & $\ldots$ & 42.6 & 44.5 & 45.0 \\
\hline Recurrent expenditures (percent of recurrent resources) & 96 & 104 & 99 & 103 & 96 & 101 & 98 & 101 & 101 \\
\hline Nominal GDP & 26,497 & 30,253 & 34,652 & 34,763 & 39,336 & 39,336 & 39,684 & 44,839 & 50,715 \\
\hline
\end{tabular}

Sources: Ministry of Finance; Bank of Tanzania; and IMF staff projections.

${ }^{1}$ Fiscal year: July-June.

${ }^{2}$ Local Government Authorities' own revenues (about 0.5 percent of GDP), and the equal amount of transfers, are included starting from FY2009/10.

${ }^{3}$ Excludes interest payments associated with external debt obligations that are under negotiation for relief with a number of creditors.

${ }^{4}$ Basket funds are sector-specific accounts established by the government to channel donor support to fund-specific activities.

${ }^{5}$ Unidentified financing (+)/expenditure (-). Includes expenditure carryover from the previous year.

${ }^{6}$ From the second review under the PSI.

${ }^{7}$ Excludes external debt under negotiation for relief and Treasury bills issued for monetary purposes. 
Table 2b. Tanzania: Central Government Operations, 2008/09-2013/14 ${ }^{1}$ (Percent of GDP)

\begin{tabular}{|c|c|c|c|c|c|c|c|c|c|}
\hline & \multirow[t]{2}{*}{$2008 / 09$} & \multirow[t]{2}{*}{$2009 / 10$} & \multicolumn{2}{|c|}{ 2010/11 } & \multicolumn{3}{|c|}{$2011 / 12$} & \multirow{2}{*}{$\frac{2012 / 13}{\text { Proj. }}$} & \multirow{2}{*}{$\begin{array}{r}\text { 2013/14 } \\
\text { Proj. }\end{array}$} \\
\hline & & & Prog. $^{6}$ & Actual & Prog. ${ }^{6}$ & Budget & Proj. & & \\
\hline Total revenue & 16.2 & 15.9 & 16.1 & 16.5 & 16.3 & 17.7 & 17.8 & 17.5 & 17.7 \\
\hline Tax revenue & 15.3 & 14.6 & 14.9 & 15.2 & 15.0 & 15.8 & 15.6 & 15.7 & 15.8 \\
\hline Import duties & 1.4 & 1.2 & 1.3 & 1.3 & 1.3 & 1.4 & 1.4 & 1.4 & 1.4 \\
\hline Value-added tax & 4.6 & 4.6 & 4.7 & 4.4 & 4.7 & 5.2 & 4.9 & 5.0 & 5.0 \\
\hline Excises & 2.9 & 2.8 & 2.6 & 3.0 & 2.6 & 2.8 & 2.7 & 2.7 & 2.8 \\
\hline Income taxes & 4.6 & 4.4 & 4.7 & 4.8 & 4.7 & 4.7 & 4.9 & 4.9 & 5.0 \\
\hline Other taxes & 1.7 & 1.7 & 1.7 & 1.7 & 1.7 & 1.7 & 1.7 & 1.7 & 1.7 \\
\hline Nontax revenue $^{2}$ & 0.9 & 1.2 & 1.2 & 1.3 & 1.3 & 1.8 & 2.2 & 1.8 & 1.9 \\
\hline LGA & & 0.5 & 0.4 & 0.3 & 0.4 & 0.9 & 0.9 & 0.9 & 1.0 \\
\hline Other & & 0.8 & 0.8 & 0.9 & 0.9 & 0.9 & 1.3 & 0.9 & 1.0 \\
\hline Total expenditure & 26.1 & 27.5 & 29.0 & 27.2 & 28.6 & 32.1 & 31.1 & 29.3 & 28.8 \\
\hline Recurrent expenditure & 17.7 & 18.8 & 18.1 & 19.2 & 17.0 & 19.6 & 19.4 & 19.4 & 19.4 \\
\hline Wages and salaries & 6.1 & 5.7 & 6.8 & 6.7 & 6.3 & 7.2 & 7.3 & 7.3 & 7.3 \\
\hline Interest payments & 0.9 & 0.8 & 0.8 & 1.0 & 1.2 & 0.8 & 1.2 & 1.1 & 1.1 \\
\hline Domestic & 0.8 & 0.7 & 0.6 & 0.8 & 0.9 & 0.6 & 0.8 & 0.7 & 0.7 \\
\hline Foreign $^{3}$ & 0.1 & 0.1 & 0.2 & 0.2 & 0.3 & 0.2 & 0.3 & 0.4 & 0.4 \\
\hline Goods and services and transfers ${ }^{2}$ & 10.7 & 12.3 & 10.4 & 11.5 & 9.5 & 11.6 & 11.0 & 11.0 & 11.0 \\
\hline Development expenditure & 8.4 & 8.6 & 10.9 & 7.9 & 11.6 & 12.5 & 11.6 & 9.9 & 9.3 \\
\hline Domestically financed & 3.4 & 3.3 & 3.9 & 2.8 & 4.0 & 4.8 & 4.3 & 3.9 & 3.6 \\
\hline Foreign (concessionally) financed & 5.0 & 5.3 & 6.9 & 5.1 & 7.6 & 7.8 & 7.3 & 6.0 & 5.7 \\
\hline Overall balance before grants & -9.9 & -11.6 & -12.9 & -10.6 & -12.4 & -14.5 & -13.2 & -11.8 & -11.0 \\
\hline Grants & 5.1 & 4.6 & 6.4 & 4.7 & 6.4 & 6.9 & 6.6 & 5.1 & 4.8 \\
\hline Program (including basket grants) ${ }^{4}$ & 3.0 & 3.1 & 3.1 & 3.1 & 2.4 & 2.8 & 3.1 & 2.7 & 2.5 \\
\hline of which: basket grants & 0.7 & 0.9 & 1.0 & 1.0 & 1.0 & 1.0 & 1.1 & 1.0 & 1.0 \\
\hline Project & 1.7 & 1.5 & 2.5 & 1.1 & 3.4 & 2.9 & 2.3 & 2.1 & 2.0 \\
\hline MDRI (IMF) grant relief & 0.3 & 0.1 & 0.0 & 0.0 & 0.0 & 0.0 & 0.0 & 0.0 & 0.0 \\
\hline MCA funding & 0.0 & 0.1 & 0.8 & 0.6 & 0.6 & 1.2 & 1.3 & 0.3 & 0.3 \\
\hline Overall balance after grants & -4.8 & -7.0 & -6.5 & -6.0 & -6.0 & -7.6 & -6.6 & -6.6 & -6.2 \\
\hline Adjustment to cash $^{5}$ & 0.2 & 0.6 & 0.0 & -0.9 & 0.0 & 0.0 & 0.0 & 0.0 & 0.0 \\
\hline Overall balance (cash basis) & -4.6 & -6.4 & -6.5 & -6.9 & -6.0 & -7.6 & -6.6 & -6.6 & -6.2 \\
\hline Unidentified fiscal measures & 0.0 & 0.0 & 0.0 & 0.0 & 0.0 & 0.0 & 0.0 & -1.1 & -1.7 \\
\hline Financing & 4.6 & 6.4 & 6.5 & 6.9 & 6.0 & 7.6 & 6.6 & 5.5 & 4.5 \\
\hline Foreign (net) & 3.6 & 4.6 & 5.4 & 3.3 & 5.0 & 6.1 & 5.6 & 4.5 & 3.5 \\
\hline Foreign loans & 3.7 & 4.8 & 5.5 & 3.4 & 5.3 & 6.3 & 5.9 & 5.1 & 4.3 \\
\hline Program (including basket loans) ${ }^{4}$ & 1.9 & 2.5 & 1.4 & 1.1 & 1.4 & 1.2 & 1.6 & 1.4 & 1.3 \\
\hline Of which: basket loans & 0.6 & 0.6 & 0.7 & 0.6 & 0.8 & 0.8 & 0.6 & 0.5 & 0.5 \\
\hline Project & 1.8 & 2.3 & 2.0 & 1.9 & 1.9 & 1.9 & 2.0 & 2.0 & 2.0 \\
\hline Nonconcessional borrowing & 0.0 & 0.0 & 2.1 & 0.4 & 2.1 & 3.2 & 2.4 & 1.7 & 1.0 \\
\hline Amortization & -0.1 & -0.2 & -0.1 & -0.1 & -0.3 & -0.2 & -0.3 & -0.5 & -0.8 \\
\hline Domestic (net) & 0.8 & 1.9 & 1.0 & 3.6 & 1.0 & 1.0 & 1.0 & 1.0 & 1.0 \\
\hline Bank financing & 0.8 & 1.9 & 0.9 & 2.6 & 0.9 & 1.0 & 0.9 & 0.9 & 0.9 \\
\hline Nonbank financing & 0.0 & -0.1 & 0.1 & 1.0 & 0.1 & 0.0 & 0.1 & 0.1 & 0.1 \\
\hline One-off transfers from agencies & 0.0 & 0.0 & 0.0 & 0.0 & 0.0 & 0.4 & 0.0 & 0.0 & 0.0 \\
\hline Privatization proceeds & 0.2 & 0.0 & 0.1 & 0.0 & 0.0 & 0.0 & 0.0 & 0.0 & 0.0 \\
\hline Amortization of parastatal debt & 0.0 & 0.0 & 0.0 & 0.0 & 0.0 & 0.0 & 0.0 & 0.0 & 0.0 \\
\hline
\end{tabular}

Sources: Ministry of Finance; Bank of Tanzania; and IMF staff projections.

${ }^{1}$ Fiscal year: July-June.

${ }^{2}$ Local Government Authorities' own revenues (about 0.5 percent of GDP), and the equal amount of transfers, are included starting from FY2009/10.

${ }^{3}$ Excludes interest payments associated with external debt obligations that are under negotiation for relief with a number of creditors.

${ }^{4}$ Basket funds are sector-specific accounts established by the government to channel donor support to fund-specific activities.

${ }^{5}$ Unidentified financing (+)/expenditure (-). Includes expenditure carryover from the previous year.

${ }^{6}$ From the second review under the PSI. 
Table 3. Tanzania: Monetary Accounts, 2008/09-2011/12

(Billions of Tanzania shillings, unless otherwise indicated; end of period)

\begin{tabular}{|c|c|c|c|c|c|c|}
\hline & \multirow[t]{2}{*}{$2008 / 09$} & \multirow[t]{2}{*}{$2009 / 10$} & \multicolumn{2}{|c|}{ 2010/11 } & \multicolumn{2}{|c|}{$2011 / 12$} \\
\hline & & & Prog. $^{2}$ & $\overline{\text { Actual }}$ & Prog. $^{2}$ & Proj. \\
\hline \multicolumn{7}{|l|}{ Bank of Tanzania } \\
\hline Net foreign assets & 3,401 & 3,949 & 4,832 & 4,618 & 5,479 & 5,380 \\
\hline Net international reserves & 3,464 & 4,336 & 5,285 & 5,098 & 5,948 & 5,880 \\
\hline (Millions of U.S. dollars) & 2,666 & 3,143 & 3,477 & 3,243 & 3,779 & 3,500 \\
\hline Net non-reserve foreign assets & -63 & -387 & -453 & -479 & -469 & -500 \\
\hline Net domestic assets & -723 & -580 & $-1,044$ & -829 & -971 & -920 \\
\hline Credit to government & -712 & -628 & -577 & -186 & -577 & -161 \\
\hline Of which: Excluding counterpart of liquidity paper & 278 & 581 & 373 & 791 & 373 & 639 \\
\hline Other items (net) & -11 & 48 & -467 & -642 & -394 & -759 \\
\hline REPOs & -131 & -117 & -30 & -54 & -93 & -24 \\
\hline Other items, excluding REPOs (net) & 120 & 165 & -437 & -588 & -487 & -735 \\
\hline Of which: Credit to nongovernment sector & 40 & 89 & 91 & 86 & 91 & 86 \\
\hline Reserve money ${ }^{1}$ & 2,678 & 3,369 & 3,789 & 3,790 & 4,508 & 4,459 \\
\hline Currency outside banks & 1,424 & 1,680 & 1,991 & 2,081 & 2,339 & 2,463 \\
\hline Bank reserves & 1,255 & 1,689 & 1,798 & 1,709 & 2,169 & 1,996 \\
\hline Currency in banks & 259 & 351 & 438 & 423 & 515 & 542 \\
\hline Deposits & 996 & 1,338 & 1,359 & 1,286 & 1,654 & 1,455 \\
\hline Required reserves (calculated) ${ }^{1}$ & 787 & 994 & 1,165 & 1,172 & 1,489 & 1,328 \\
\hline Excess reserves (calculated) & 209 & 344 & 194 & 114 & 165 & 127 \\
\hline \multicolumn{7}{|l|}{ Memorandum items: } \\
\hline Stock of liquidity paper & 990 & 1,209 & 950 & 977 & 950 & 800 \\
\hline Average reserve money & 2,602 & 3,138 & 3,766 & 3,746 & 4,482 & 4,420 \\
\hline \multicolumn{7}{|l|}{ Monetary Survey } \\
\hline Net foreign assets & 4,203 & 5,266 & 6,273 & 5,804 & 6,908 & 6,728 \\
\hline Bank of Tanzania & 3,401 & 3,949 & 4,832 & 4,618 & 5,479 & 5,380 \\
\hline Commercial banks & 802 & 1,316 & 1,441 & 1,186 & 1,429 & 1,348 \\
\hline Net domestic assets & 3,629 & 4,536 & 5,496 & 6,154 & 7,097 & 7,626 \\
\hline Domestic credit & 4,580 & 5,908 & 7,401 & 8,126 & 9,086 & 9,980 \\
\hline Credit to government (net) & 128 & 713 & 1,010 & 1,620 & 1,363 & 1,977 \\
\hline Credit to nongovernment sector & 4,452 & 5,195 & 6,391 & 6,506 & 7,723 & 8,003 \\
\hline Other items (net) & -951 & $-1,372$ & $-1,905$ & $-1,972$ & $-1,989$ & $-2,354$ \\
\hline M3 & 7,832 & 9,801 & 11,769 & 11,958 & 14,005 & 14,353 \\
\hline Foreign currency deposits & 2,064 & 2,522 & 3,295 & 3,291 & 3,922 & 4,091 \\
\hline M2 & 5,768 & 7,279 & 8,474 & 8,667 & 10,083 & 10,263 \\
\hline Currency in circulation & 1,424 & 1,680 & 1,991 & 2,081 & 2,339 & 2,463 \\
\hline Deposits (TSh) & 4,344 & 5,599 & 6,483 & 6,586 & 7,744 & 7,800 \\
\hline Memorandum items: & \multicolumn{6}{|c|}{ (12-month percent change, unless otherwise indicated) } \\
\hline M3 growth & 18.5 & 25.1 & 20.1 & 22.0 & 19.0 & 20.0 \\
\hline M3 (as percent of GDP) & 29.6 & 32.4 & 34.0 & 34.4 & 35.6 & 36.2 \\
\hline Private sector credit growth & 33.1 & 16.7 & 23.0 & 25.2 & 20.8 & 23.0 \\
\hline Average reserve money & 26.7 & 20.6 & 20.0 & 19.3 & 19.0 & 18.0 \\
\hline Reserve money multiplier (M3/average reserve money) & 3.0 & 3.1 & 3.1 & 3.2 & 3.1 & 3.2 \\
\hline
\end{tabular}

Sources: Bank of Tanzania and IMF staff estimates and projections.

${ }^{1}$ In January 2009 the reserve requirement on government deposits was increased to 20 percent (from 10 percent) and cash in banks was no longer counted towards required reserves. Previously, 50 percent of cash in banks was counted toward required reserves.

${ }^{2}$ From the second review under the PSI. 
Table 4. Tanzania: Financial Soundness Indicators, 2007-2011

(Percent, end of period)

\begin{tabular}{|c|c|c|c|c|c|c|c|c|c|c|}
\hline & \multirow[b]{2}{*}{2007} & \multirow[b]{2}{*}{2008} & \multirow[b]{2}{*}{2009} & \multicolumn{4}{|c|}{2010} & \multicolumn{3}{|c|}{2011} \\
\hline & & & & Mar & Jun & Sep & Dec & Mar & Jun & Sep \\
\hline \multicolumn{11}{|l|}{ Capital adequacy } \\
\hline Capital to risk-weighted assets & 16.2 & 17.0 & 18.3 & 20.5 & 19.4 & 19.3 & 18.9 & 19.2 & 18.2 & 17.4 \\
\hline Capital to assets & 8.1 & 10.1 & 10.9 & 11.9 & 11.0 & 11.1 & 10.9 & 11.2 & 10.6 & 10.4 \\
\hline \multicolumn{11}{|l|}{ Asset composition and quality } \\
\hline Net loans and advances to total assets & 41.2 & 50.6 & 46.3 & 45.2 & 44.8 & 44.6 & 44.1 & 44.8 & 45.9 & 47.3 \\
\hline \multicolumn{11}{|l|}{ Sectoral distribution of loans } \\
\hline Trade & 17.0 & 18.5 & 18.8 & 18.3 & 18.0 & 17.2 & 17.5 & 18.6 & 19.4 & 20.0 \\
\hline Mining and manufacturing & 20.2 & 14.7 & 12.0 & 13.3 & 15.4 & 15.8 & 14.2 & 14.0 & 13.3 & 13.4 \\
\hline Agricultural production & 11.9 & 10.4 & 10.2 & 10.2 & 11.3 & 13.3 & 13.1 & 13.1 & 13.2 & 14.3 \\
\hline Building and construction & 5.1 & 4.9 & 5.0 & 4.9 & 5.6 & 7.0 & 6.1 & 7.7 & 7.4 & 7.8 \\
\hline Transport and communication & 6.9 & 7.3 & 9.2 & 8.9 & 10.1 & 9.3 & 9.2 & 9.5 & 8.5 & 7.8 \\
\hline Foreign exchange loans to total loans & 31.4 & 31.8 & 28.5 & 29.1 & 32.1 & 32.9 & 32.0 & 32.6 & 34.6 & 35.3 \\
\hline Gross nonperforming loans (NPLs) to gross loans & 6.3 & 6.2 & 6.6 & 7.0 & 7.2 & 7.9 & 9.3 & 9.6 & 9.1 & 8.1 \\
\hline NPLs net of provisions to capital & 22.0 & 22.0 & 17.6 & 15.4 & 17.3 & 19.4 & 24.3 & 24.6 & 25.4 & 21.7 \\
\hline Large exposures to total capital & 183.5 & 199.4 & 54.4 & 93.7 & 161.5 & 98.5 & 104.2 & 95.0 & 110.9 & 122.3 \\
\hline \multicolumn{11}{|l|}{ Earnings and profitability } \\
\hline Return on assets & 4.7 & 3.8 & 3.2 & 2.7 & 2.9 & 2.9 & 2.3 & 3.0 & 3.0 & 3.0 \\
\hline Return on equity & 29.0 & 23.2 & 18.4 & 15.3 & 16.4 & 16.1 & 12.1 & 17.8 & 17.9 & 17.2 \\
\hline Net interest margin & 73.8 & 76.7 & 73.3 & 73.2 & 74.8 & 75.1 & 75.8 & 77.6 & 78.5 & 78.5 \\
\hline Noninterest expenses to gross income & 42.5 & 48.8 & 47.6 & 53.5 & 52.5 & 52.9 & 54.5 & 52.1 & 53.7 & 54.5 \\
\hline Personnel expenses to noninterest expenses & 40.5 & 40.6 & 42.6 & 43.6 & 42.5 & 41.5 & 40.5 & 44.4 & 43.8 & 42.7 \\
\hline \multicolumn{11}{|l|}{ Liquidity } \\
\hline Liquid assets to total assets & 48.0 & 37.6 & 40.5 & 40.5 & 40.8 & 39.7 & 40.4 & 38.8 & 38.2 & 37.6 \\
\hline Liquid assets to total short term liabilities & 53.0 & 41.7 & 46.5 & 47.1 & 46.9 & 45.6 & 46.1 & 44.3 & 43.2 & 41.8 \\
\hline Total loans to customer deposits & 57.6 & 68.4 & 61.6 & 60.0 & 59.1 & 59.6 & 58.9 & 59.6 & 61.5 & 63.0 \\
\hline Foreign exchange liabilities to total liabilities & 33.8 & 32.5 & 29.7 & 29.1 & 30.5 & 32.0 & 31.8 & 33.3 & 33.9 & 36.0 \\
\hline
\end{tabular}

Source: Bank of Tanzania 
Table 5. Tanzania: Summary Accounts of the Bank of Tanzania, 2011-12 (Billions of Tanzania shillings, unless otherwise indicated; end of period)

\begin{tabular}{|c|c|c|c|c|c|c|}
\hline & \multicolumn{4}{|c|}{2011} & \multicolumn{2}{|c|}{2012} \\
\hline & Mar & Jun & Sep & $\overline{\mathrm{Dec}}$ & Mar & Jun \\
\hline & Act. & Act. & Act. & Proj. & Proj. & Proj. \\
\hline Net foreign assets & 4,515 & 4,618 & 4,601 & 5,075 & 5,246 & 5,380 \\
\hline Net international reserves & 4,963 & 5,098 & 5,087 & 5,575 & 5,746 & 5,880 \\
\hline (Millions of U.S. dollars) & 3,329 & 3,243 & 3,119 & 3,322 & 3,423 & 3,500 \\
\hline Net non-reserve foreign assets & -447 & -479 & -486 & -500 & -500 & -500 \\
\hline Net domestic assets & -961 & -829 & -362 & -868 & -945 & -920 \\
\hline Credit to government & -484 & -186 & 369 & -181 & -231 & -161 \\
\hline Of which: Excluding counterpart of liquidity paper & 589 & 791 & 853 & 469 & 569 & 639 \\
\hline Other items (net) & -477 & -642 & -731 & -687 & -713 & -759 \\
\hline REPOs & -85 & -54 & -28 & 42 & 19 & -24 \\
\hline Other items, excluding REPOs (net) & -393 & -588 & -703 & -729 & -732 & -735 \\
\hline Of which: Credit to nongovernment sector & 90 & 86 & 86 & 86 & 86 & 86 \\
\hline Reserve money ${ }^{1}$ & 3,554 & 3,790 & 4,239 & 4,207 & 4,302 & 4,459 \\
\hline Currency outside banks & 1,855 & 2,081 & 2,243 & 2,295 & 2,247 & 2,463 \\
\hline Bank reserves & 1,699 & 1,709 & 1,996 & 1,912 & 2,055 & 1,996 \\
\hline Currency in banks & 410 & 423 & 419 & 505 & 494 & 542 \\
\hline Deposits & 1,289 & 1,286 & 1,577 & 1,407 & 1,560 & 1,455 \\
\hline Required reserves (calculated) & 1,104 & 1,172 & 1,232 & 1,296 & 1,434 & 1,328 \\
\hline Excess reserves (calculated) & 185 & 114 & 345 & 112 & 126 & 127 \\
\hline \multicolumn{7}{|l|}{ Memorandum items: } \\
\hline Stock of liquidity paper & 1,073 & 977 & 484 & 650 & 800 & 800 \\
\hline Average reserve money & 3,625 & 3,746 & 4,095 & 4,193 & 4,278 & 4,420 \\
\hline
\end{tabular}

Sources: Bank of Tanzania and IMF staff estimates and projections. 
Table 6. Tanzania: Monetary Survey, 2011-12 (Billions of Tanzania shillings, unless otherwise indicated; end of period)

\begin{tabular}{|c|c|c|c|c|c|c|}
\hline & \multicolumn{4}{|c|}{2011} & \multicolumn{2}{|c|}{2012} \\
\hline & Mar & Jun & Sep & $\overline{\mathrm{Dec}}$ & Mar & Jun \\
\hline & Act. & Act. & Act. & Proj. & Proj. & Proj. \\
\hline Net foreign assets & 5,805 & 5,804 & 6,017 & 6,514 & 6,602 & 6,728 \\
\hline Bank of Tanzania & 4,515 & 4,618 & 4,601 & 5,075 & 5,246 & 5,380 \\
\hline Commercial banks & 1,290 & 1,186 & 1,415 & 1,439 & 1,356 & 1,348 \\
\hline Net domestic assets & 5,429 & 6,154 & 6,784 & 6,842 & 7,039 & 7,626 \\
\hline Domestic credit & 7,246 & 8,126 & 8,959 & 9,089 & 9,390 & 9,980 \\
\hline Credit to government (net) & 1,227 & 1,620 & 1,927 & 1,899 & 1,885 & 1,977 \\
\hline Credit to nongovernment sector & 6,018 & 6,506 & 7,031 & 7,190 & 7,504 & 8,003 \\
\hline Other items (net) & $-1,816$ & $-1,972$ & $-2,174$ & $-2,247$ & $-2,351$ & $-2,354$ \\
\hline M3 & 11,234 & 11,958 & 12,801 & 13,357 & 13,641 & 14,353 \\
\hline Foreign currency deposits & 3,097 & 3,291 & 3,717 & 3,873 & 3,956 & 4,091 \\
\hline M2 & 8,137 & 8,667 & 9,084 & 9,483 & 9,685 & 10,263 \\
\hline Currency in circulation & 1,855 & 2,081 & 2,243 & 2,295 & 2,247 & 2,463 \\
\hline Deposits (Tanzania Sh) & 6,282 & 6,586 & 6,841 & 7,188 & 7,438 & 7,800 \\
\hline \multicolumn{7}{|l|}{ Memorandum items: } \\
\hline M3 growth (12-month percent change) & 23.8 & 22.0 & 23.7 & 21.3 & 21.4 & 20.0 \\
\hline Credit to private sector (12-month percent change) & 24.0 & 25.2 & 29.6 & 25.7 & 24.7 & 23.0 \\
\hline Average reserve money (12-month percent change) & 19.8 & 19.3 & 18.7 & 19.0 & 18.0 & 18.0 \\
\hline Nonbank financing of the government (net) ${ }^{1}$ & 173 & 337 & -4 & 20 & 30 & 40 \\
\hline Bank financing of the government (net) ${ }^{1}$ & 515 & 907 & 307 & 280 & 266 & 357 \\
\hline Bank and nonbank financing of the government (net) ${ }^{1}$ & 687 & 1,244 & 304 & 300 & 296 & 397 \\
\hline
\end{tabular}

Sources: Bank of Tanzania and IMF staff estimates and projections.

${ }^{1}$ Cumulative from the beginning of the fiscal year (July 1 ). 
Table 7. Tanzania: Balance of Payments, 2008/09-2013/14

(Millions of U.S. dollars, unless otherwise indicated)

\begin{tabular}{|c|c|c|c|c|c|c|c|c|}
\hline & \multirow[t]{2}{*}{ 2008/09 } & \multirow[t]{2}{*}{ 2009/10 } & \multicolumn{2}{|c|}{ 2010/11 } & \multicolumn{2}{|c|}{$2011 / 12$} & \multirow{2}{*}{$\begin{array}{r}2012 / 13 \\
\text { Proj. }\end{array}$} & \multirow{2}{*}{$\begin{array}{r}2013 / 14 \\
\text { Proj. }\end{array}$} \\
\hline & & & Prog. ${ }^{4}$ & Prel. & Prog. $^{4}$ & Proj. & & \\
\hline Current account & $-2,238$ & $-2,085$ & $-2,207^{r}$ & $-2,283$ & $-2,719$ & $-2,399$ & $-2,558$ & $-2,577$ \\
\hline Trade balance & $-2,952$ & $-2,771$ & $-3,190^{\prime}$ & $-3,123$ & $-3,562$ & $-3,174$ & $-3,386$ & $-3,431$ \\
\hline $\begin{array}{c}\text { Exports, f.o.b. } \\
\text { Of which }\end{array}$ & 3,268 & 3,799 & 4,683 & 4,888 & 5,188 & 5,865 & 6,648 & 7,499 \\
\hline Traditional agricultural products & 510 & 457 & 610 & 693 & 518 & 832 & 942 & 1,072 \\
\hline Gold & 925 & 1,493 & 1,668 & 1,732 & 2,097 & 2,216 & 2,568 & 2,850 \\
\hline Imports, f.o.b & $-6,220$ & $-6,570$ & $-7,873$ & $-8,012$ & $-8,750$ & $-9,039$ & $-10,034$ & $-10,931$ \\
\hline Of which: Oil & $-1,485$ & $-1,911$ & $-2,368$ & $-2,114$ & $-2,642$ & $-2,466$ & $-2,660$ & $-2,839$ \\
\hline Services (net) & 163 & 173 & 239 & 271 & 266 & 336 & 459 & 558 \\
\hline Of which: Travel receipts & 1,163 & 1,197 & 1,359 & 1,357 & 1,514 & 1,524 & 1,662 & 1,816 \\
\hline Income (net) & -183 & -148 & -33 & -190 & -71 & -290 & -374 & -461 \\
\hline Of which: interest on public debt & -28 & -31 & -46 & -46 & -82 & -74 & -108 & -122 \\
\hline Current transfers (net) & 734 & 660 & 777 & 759 & 648 & 729 & 743 & 758 \\
\hline Of which: official transfers & 703 & 623 & 736 & 737 & 606 & 691 & 705 & 719 \\
\hline Capital account & 379 & 608 & 611 & 560 & 929 & 616 & 631 & 648 \\
\hline Of which: project grants ${ }^{1}$ & 317 & 545 & 579 & 496 & 865 & 547 & 558 & 569 \\
\hline Financial account & 1,306 & 1,627 & $1,931^{r}$ & 1,632 & 2,095 & 2,043 & 2,139 & 2,129 \\
\hline Foreign Direct Investment & 408 & 424 & 442 & 444 & 542 & 542 & 693 & 893 \\
\hline $\begin{array}{l}\text { Public Sector, net } \\
\text { Of which: }\end{array}$ & 719 & 1,058 & 1,274 & 799 & 1,274 & 1,343 & 1,195 & 1,002 \\
\hline Program loans & 400 & 612 & 322 & 267 & 352 & 370 & 366 & 373 \\
\hline Non-concessional borrowing & 0 & 0 & 525 & 85 & 525 & 575 & 450 & 289 \\
\hline Project loans & 387 & 520 & 455 & 475 & 479 & 469 & 515 & 567 \\
\hline Scheduled amortization ${ }^{2}$ & -21 & -29 & -29 & -28 & -82 & -70 & -136 & -226 \\
\hline Commercial Banks, net & -9 & -320 & -4 & 202 & 40 & -61 & 40 & 10 \\
\hline SDR allocation ${ }^{3}$ & 0 & 249 & 0 & 0 & 0 & 0 & 0 & 0 \\
\hline Other private inflows & 188 & 216 & 219 & 188 & 239 & 219 & 210 & 223 \\
\hline Errors and omissions & 571 & 328 & 0 & 190 & 0 & 0 & 0 & 0 \\
\hline Overall balance & 18 & 478 & $335^{\prime}$ & 100 & 305 & 260 & 212 & 200 \\
\hline Financing & -18 & -478 & $-335^{r}$ & -100 & -305 & -260 & -212 & -200 \\
\hline Change in BoT reserve assets (increase $=-$ ) & -264 & -570 & -334 & -128 & -302 & -258 & -208 & -184 \\
\hline Use of Fund credit & 246 & 93 & -1 & 29 & -3 & -3 & -3 & -16 \\
\hline Financing gap & 0 & 0 & 0 & 0 & 0 & 0 & 0 & 0 \\
\hline \multicolumn{9}{|l|}{ Memorandum items: } \\
\hline Gross official reserves (BoT) & 2,930 & 3,483 & 3,831 & 3,610 & 4,133 & 3,868 & 4,076 & 4,260 \\
\hline Months of imports of goods and services (current year) & 4.5 & 5.0 & 4.7 & 4.4 & 4.5 & 4.1 & 4.0 & 3.8 \\
\hline Exports (percent of GDP) & 15.6 & 16.8 & 20.1 & 21.0 & 20.4 & 24.5 & 25.3 & 26.0 \\
\hline Imports (percent of GDP) & -29.7 & -29.0 & -33.8 & -34.4 & -34.4 & -37.8 & -38.2 & -37.9 \\
\hline Current account deficit (percent of GDP) & -10.7 & -9.2 & -9.5 & -9.8 & -10.7 & -10.0 & -9.7 & -8.9 \\
\hline Foreign program and project assistance (percent of GDP) & 8.6 & 10.2 & 9.0 & 8.5 & 9.1 & 8.7 & 8.2 & 7.7 \\
\hline Nominal GDP & 20,956 & 22,620 & 23,295 & 23,321 & 25,428 & 23,909 & 26,299 & 28,875 \\
\hline
\end{tabular}

Sources: Tanzanian authorities and IMF staff estimates and projections.

${ }^{1}$ An adjustment to the estimated outturn is made by BoT to reflect unreported project grants.

${ }^{2}$ Relief on some projected external debt obligations is being negotiated with a number of creditors.

${ }^{3}$ In 2009, Tanzania received SDR 147.4 million on August 28 and SDR 11.7 million on September 9 (equivalent to US\$249 million in total).

${ }^{4}$ From the second review under the PSI. 
Table 8. Tanzania: Quantitative Assessment Criteria Under the Policy Support Instrument, June and September 2011

\begin{tabular}{|c|c|c|c|c|c|c|c|c|}
\hline & \multicolumn{4}{|c|}{ June } & \multicolumn{4}{|c|}{ September } \\
\hline & $\begin{array}{l}\text { Assessment } \\
\text { Criteria }\end{array}$ & $\begin{array}{l}\text { Adjusted } \\
\text { Criteria }\end{array}$ & Actual & Met? & $\begin{array}{l}\text { Indicative } \\
\text { Target }\end{array}$ & $\begin{array}{l}\text { Adjusted } \\
\text { Targets }\end{array}$ & Actual & Met? \\
\hline & \multicolumn{8}{|c|}{ (Billions of Tanzania Shillings; end of period, unless otherwise indicated) } \\
\hline $\begin{array}{l}\text { Net domestic financing of the government of Tanzania } \\
\text { (cumulative, ceiling) }{ }^{1,2}\end{array}$ & 347 & 431 & 1244 & $\mathbf{x}$ & 122 & 372 & 304 & $\checkmark$ \\
\hline Average reserve money (upper bound) ${ }^{3}$ & 3,804 & 3,804 & 3,746 & $\checkmark$ & 4,147 & 4,147 & 4,095 & $\checkmark$ \\
\hline Average reserve money target ${ }^{3}$ & 3,766 & & & & 4,106 & & & \\
\hline Average reserve money (lower bound) ${ }^{3}$ & 3,729 & & & & 4,065 & & & \\
\hline & \multicolumn{8}{|c|}{ (Millions of U.S. dollars; end of period) } \\
\hline Net international reserves of the Bank of Tanzania (floor) ${ }^{4}$ & 3,477 & 3,421 & 3,243 & $\mathbf{x}$ & 3,622 & 3,458 & 3,119 & $\mathbf{x}$ \\
\hline Accumulation of external payment arrears (ceiling) & 0 & 0 & 0 & $\checkmark$ & 0 & 0 & 0 & $\checkmark$ \\
\hline $\begin{array}{l}\text { Contracting or guaranteeing of external debt on } \\
\text { nonconcessional terms (continuous ceiling) }\end{array}$ & 525 & 525 & 103 & $\checkmark$ & 525 & 525 & 250 & $\checkmark$ \\
\hline \multicolumn{9}{|l|}{ Memorandum item: } \\
\hline Foreign program assistance (cumulative grants and loan & 1,058 & 1,058 & 1,002 & & 299 & 299 & 24 & \\
\hline
\end{tabular}

Note: For precise definitions of the aggregates shown and details of the adjustment clauses, see the Technical Memorandum of Understanding (TMU).

${ }^{1}$ Cumulative from the beginning of the fiscal year (July 1 ).

2 To be adjusted upward by up to TSh 250 billion for the U.S. dollar equivalent of a shortfall in foreign program assistance from the amounts shown in the

memorandum item.

${ }^{3}$ Assessment criteria and benchmarks apply to upper bound only.

${ }^{4}$ Floor will be adjusted downward for any shortfall in foreign program assistance from the amounts shown in the memorandum item up to the equivalent of TSh 250 billion.

${ }^{5}$ To be used exclusively for infrastructure investment projects. Continuous assessment criteria; ceiling applies throughout the fiscal year. 
Table 9. Tanzania: Structural Benchmarks for 2011/12

\begin{tabular}{|c|c|c|}
\hline Measure & Target Date & Status \\
\hline \multicolumn{3}{|l|}{ Poverty Reduction Strategy } \\
\hline $\begin{array}{l}\text { Approval by Government of a new National Social } \\
\text { Protection Framework (NSPF). }\end{array}$ & End-June, 2011 & $\begin{array}{l}\text { Proposed to be dropped. } \\
\text { Work on a NSPF has been } \\
\text { integrated into an ongoing, } \\
\text { broader, national social } \\
\text { security reform program. }\end{array}$ \\
\hline \multicolumn{3}{|l|}{ Financial Sector } \\
\hline Issuance of investment guidelines for pension funds. & $\begin{array}{l}\text { End-September, } \\
\qquad 2011\end{array}$ & $\begin{array}{l}\text { Proposed delayed to end- } \\
\text { March } 2012 \text { to complete an } \\
\text { actuarial assessment. }\end{array}$ \\
\hline \multicolumn{3}{|l|}{ Fiscal } \\
\hline $\begin{array}{l}\text { Debt management: Prepare a Medium-Term Debt } \\
\text { Strategy consistent with resource needs under the new } \\
\text { PRS and reflecting contingent liabilities. }\end{array}$ & End-June, 2011 & Met. \\
\hline $\begin{array}{l}\text { Establish a unified public debt statistics database } \\
\text { under a single monitoring unit. }\end{array}$ & $\begin{array}{l}\text { End-December, } \\
\qquad 2011\end{array}$ & Met. \\
\hline $\begin{array}{l}\text { Develop an index for monitoring priority social } \\
\text { spending. }\end{array}$ & $\begin{array}{l}\text { End-December, } \\
\qquad 2011\end{array}$ & Met. \\
\hline
\end{tabular}




\section{LETTER OF INTENT}

Dar es Salaam

December 23, 2011

Ms. Christine Lagarde

Managing Director

International Monetary Fund

Washington, D.C. 20431

U.S.A.

Dear Madam Lagarde:

1. The Government of Tanzania remains committed to policies that will sustain macroeconomic stability while promoting accelerated economic growth and poverty reduction. These goals are being successfully pursued under the economic and financial programme supported by the Policy Support Instrument (PSI).

2. In the period through end-June 2011, the Government met three PSI quantitative assessment criteria while two criteria were missed due to temporary costs incurred in clearing part of the expenditure float in the budget and delays in contracting external nonconcessional borrowing. At the same time, progress has been made in implementing the structural reform program, with three structural benchmarks met and two planned for implementation, with a short unavoidable delay, by March 2012.

3. Tanzania's economy continues to grow strongly. Despite power shortages, real GDP grew by 6.3 percent in the first half of 2011, and most leading indicators remain favorable. Accordingly, the Government is strongly of the view that the revised 6.0 percent GDP growth projection for 2011 will be achieved and most likely surpassed. Growth is projected to increase to 7.2 percent in 2012 and remain above this level in the medium term.

4. Inflation has risen on account of the pass through from higher global oil prices, a regional surge in food prices, and a power tariff increase in January 2011. Inflation is projected to peak around October levels of 17.9 percent and remain at double digit level, before declining to around 9 percent by June 2012. These projections assume good rainfall and favorable harvests in early 2012.

5. The Government's expenditure policies continue to be guided by the National Strategy for Growth and Reduction of Poverty (MKUKUTA II), which focuses on improving the livelihoods of Tanzanians. The efficiency of resource utilisation and service delivery is being promoted by core programmes covering public financial management 
reform, public service reform, local government reform, legal sector reform, and a national anti-corruption strategy. The Government is also implementing a roadmap for improving the business climate.

6. Following discussions and consultations with the Fund staff, I hereby transmit this Letter of Intent and the attached Memorandum of Economic and Financial Policies, which reviews the implementation of the programme in 2010/11 and the first quarter of 2011/12 and policies that the Government intends to pursue this year and beyond.

7. The Government of Tanzania requests waivers for the two missed assessment criteria and completion of the third review under the PSI based on overall performance under the program and the Government's policy intentions going forward. The Government is confident that the policies set forth in the attached MEFP are adequate to achieve the objectives of its programme, and following further consultation with the Fund, will take any appropriate measures for this purpose. The Government will regularly update the Fund on developments in its economic and financial policies, and will provide the data needed for monitoring the programme. The Government will consult regularly with the Fund on any relevant developments at the initiative of the Government or the Fund.

8. The Government of Tanzania intends to disseminate this letter and the attached MEFP, as well as related Fund staff reports, and hereby authorizes the IMF to publish the same on its website after consideration by the Executive Board.

Yours Sincerely, $/ \mathrm{s} /$

Mustafa H. Mkulo

MINISTER FOR FINANCE

UNITED REPUBLIC OF TANZANIA

Attachments:

Memorandum of Economic and Financial Policies

Technical Memorandum of Understanding 


\title{
Attachment I. Memorandum of Economic and Financial Policies
}

\author{
December 23, 2011
}

\section{Recent Macroeconomic Developments}

1. Tanzania's macroeconomic performance remained strong, despite shortages of power from hydroelectric generators. Growth was 6.3 percent in the first half of 2011, down only modestly from 7.4 percent in the corresponding period in 2010. Double-digit growth was achieved in construction (16.3 percent), transport and communication (11.7 percent), and financial intermediation (11.1 percent). Based on performance in the first half of 2011, the full year GDP growth projection of 6.0 percent is likely to be achieved, and possibly exceeded.

2. Inflation has risen from 4.2 percent in October 2010 to 17.9 percent in October 2011 reflecting drought-based regional food supply shortages, high global fuel prices, and an increase in domestic power tariffs in January 2011. Core inflation remained in single digits, at 8.5 percent in October 2011.

3. In 2010/11, total revenues performed well, rising 19.6 percent from 2009/10 levels and exceeding PSI projections by 0.4 percent of GDP; this reflected favorable implementation of the revenue measures outlined in the November 2010 MEFP. However, combined grant and foreign loan financing fell 3.8 percent of GDP below PSI projections. Responding to this funding shortfall, expenditures were kept 1.8 percent of GDP below PSI projections, and domestic arrears equivalent to about 1 percent of GDP were carried over into 2011/12, primarily in regard to road infrastructures. Recurrent spending exceeded PSI projections by 1.2 percent of GDP reflecting difficulties in making the sizeable spending cuts targeted under the PSI, in relation to GDP. Expenditure savings were partly offset by unbudgeted costs to reduce the expenditure float amounting to 0.9 percent of GDP. The overall deficit of 6.9 percent of GDP was only slightly above the PSI program goal of 6.5 percent of GDP.

4. For 2011/12, revenue collections in the first quarter were favorable, broadly in line with budget goals for the full year. At the same time, donor funding started the year unusually slowly, with no general budget support and overall receipts equivalent to just 7 percent of full-year donor commitments. Most donors are expected to disburse in the second and third quarters of the fiscal year. Expenditures in the first quarter were kept in careful check, with development expenditures focused on repaying arrears carried over from 2010/11.

5. Reserve money remained on track in 2010/11 and through the first quarter of 2011/12, while broader liquidity aggregates slightly exceeded program projections. At end-September 2011, broad money supply (M3) recorded a growth rate of 23.7 percent compared with the projected rate of 19.8 percent under the PSI. Similarly, credit to the private sector grew 
somewhat faster than projected under the PSI program through September 2011, partly mirrored in a decline in the net foreign assets (NFA) and excess reserves of banks. Monetary policies have been tightened in recent months to address risks to inflation, and the weighted average Treasury bill yield increased from less than 5 percent in June 2011 to 15.16 percent in November.

6. In 2010/11, the external current account deficit (excluding official transfers) widened to 13 percent of GDP, compared with 12 percent of GDP in 2009/10. Imports of goods and services rose by 19.4 percent (compared to 5.8 percent in the preceding year), reflecting higher global oil prices and a surge in imports of capital goods. Export growth was also strong (23.8 percent, compared to 12.8 percent in 2009/10), reflecting strong performance of traditional and manufactured exports and favorable commodity export prices, particularly for gold. At end-June 2011, gross international reserves were $\$ 3,610$ million, equivalent to 4.4 months of projected imports of goods and services. At end-September 2011, reserves fell to $\$ 3,473$ million (3.7 months of projected import cover), reflecting in part delays in donor financing and non-concessional borrowing, as well as negative valuation effects on reserves held in Euros and sterling when these currencies depreciated against the dollar.

7. The exchange rate depreciated by 11.2 percent against the dollar through endNovember 2011. This partly reflected demand for foreign currency to pay oil import bills inflated by high global prices and demand linked to back-up generators operated by businesses and households during load-shedding.

8. Tanzania's public and private external debt rose from $\$ 6.5$ billion at end-June 2010 to $\$ 7.7$ billion at end-June 2011 (33 percent of GDP). Over the same period, total public debt (domestic and external) increased from 33 to 38 percent of GDP.

\section{Performance under the PSI program through September 2011}

\section{Quantitative targets}

9. Three of the five June 2011 PSI assessment criteria were met, while two were missed:

- $\quad$ The reserve money ceiling was met with a margin of 1.5 percent (TSh 58.4 billion);

- Contracting of external non-concessional debt of $\$ 103$ million was well below the relevant PSI ceiling ( $\$ 525$ million);

- The continuous criterion on the non-accumulation of external payments arrears was also met;

- Net international reserves (NIR) were \$178 million below the adjusted program floor, reflecting the shortfall in external non-concessional borrowing relative to program plans noted above; and

- $\quad$ Net domestic financing of the budget was TSh 813 billion higher than the adjusted program ceiling. This reflected the shortfall in non-concessional borrowing noted 
above, as well as improved procedures for government payments which resulted in a TSh 280 billion reduction in the expenditure float that had to be financed.

10. For September 2011, four out of five indicative targets were met. Average reserve money and net domestic financing were below their respective ceilings by TSh 52 billion and TSh 68 billion, respectively. The Government remained within the $\$ 525$ million continuous ceiling on non-concessional debt through October, signing loan agreements for $\$ 350$ million (with a potential to increase to $\$ 410$ million depending on market response during syndication); no external arrears were incurred. The adjusted NIR target was missed by $\$ 340$ million at end-September, with about half of the shortfall being a carry-over from the corresponding shortfall in June 2011, the remainder reflecting payments to clear domestic arrears to foreign contractors, unexpectedly high government imports of fuel for thermal power generation, and revaluation losses due to bilateral currency movements against the dollar. The government requests a modification of the assessment criterion on NIR from measuring NIR on a stock basis to a flow basis to eliminate base effects from performance measurement.

\section{Structural benchmarks}

11. Progress has been made in implementing the structural reform program, with three benchmarks met and two unavoidably delayed to March 2012. A medium-term debt strategy was prepared in January 2011 and published in June 2011 as scheduled; a unified debt database was established within the Ministry of Finance ahead of the end-December deadline; and the Government has developed a framework based on MKUKUTA II that permits monitoring various dimensions of social spending. Investment guidelines for pension funds have been developed, but reflecting the need to complete a preliminary actuarial assessment, will be issued with a delay by end-March 2012. Work on a National Social Protection Framework (NSPF) has been integrated into an ongoing, broader national social security reform program. This reform involves multiple institutions and the timing for approval is currently uncertain. Since Tanzania maintains an existing social protection system, and development of a more comprehensive social protection framework would not entail immediate changes on the ground, the Government requests that this benchmark be dropped.

\section{Economic Program for 2011/12 and the Medium Term}

\section{Economic prospects}

12. Economic performance is expected to strengthen further in 2012 and over the medium term, benefitting from implementation of MKUKUTA II, the Five Year Development Plan, and initiatives under the Southern Agricultural Corridor of Tanzania (SAGCOT). Reflecting favorable weather, GDP growth is projected at 7.2 percent in 2012, with somewhat higher growth over the medium term.

13. With food scarcity in the Eastern Africa region, high oil prices, and recent exchange rate depreciation, inflation is projected at about 15 percent in December 2011. Assuming good short-rains and favorable harvests in the first quarter of 2012, inflation is projected to 
ease to single digits by June 2012. Inflation would subsequently be kept in single digits by tight fiscal and monetary policies.

14. In 2011/12, the external current account deficit (excluding official transfers) is projected to remain at 13.0 percent of GDP. Over the medium term, the current account deficit is projected to return to an average of 11 percent of GDP as fiscal tightening slows import growth relative to exports.

15. The Government will continue to enhance efficiency in resource utilisation and service delivery by structural reforms in the following key areas: public financial management reform, public service reform, local government reform, legal sector reform, and a national anti-corruption strategy. In addition, the Government is supporting private sector led growth through a roadmap for improving the business climate: this roadmap has identified several "quick wins" and short-term actions that the Government intends to implement.

\section{Fiscal policy for 2011/12}

16. The 2011/12 budget envisaged total expenditure of 32.1 percent of GDP targeted on MKUKUTA II goals, up from an outturn of 27.2 percent of GDP a year earlier. This included a rise in the development spending to 12.5 percent of GDP (up from 7.9 percent of GDP a year earlier), together with recurrent spending equivalent to 19.6 percent of GDP (up from 19.3 percent of GDP a year earlier). Although the higher spending was partly offset by a projected rise in revenues and grants in relation to GDP, the budgeted fiscal deficit for 2011/12 was 7.6 percent of GDP, up from an outturn of 6.9 percent of GDP a year earlier.

17. Given the importance of safeguarding Tanzania's reputation for prudent fiscal management, and in light of the less favorable global financing situation, the Government has decided to adopt mid-year measures to reduce the 2011/12 deficit to 6.6 percent of GDP (compared to an earlier 2011/12 deficit goal under the PSI of 6.0 percent of GDP). A broadbased range of expenditure savings have been identified through a line by line review of the budget. These have been approved by the Government, and are being reflected in cash ceilings for the remainder of 2011/12. Specifically:

- $\quad$ Savings in goods and services and transfers will amount to TSh 203 billion (0.5 percent of GDP). Cuts are being made, without serious adverse economic impact, to extra duty allowances, training, travel, office running costs, vehicle operating costs, allocations to government agencies and boards, and some activities of local government authorities (LGAs) and new administrative areas;

- $\quad$ Savings in domestically-financed development expenditures will amount to TSh 157 billion ( 0.4 percent of GDP). A number of construction and rehabilitation projects have been identified that can be slowed without incurring penalties; and

- $\quad$ Additional revenues will amount to TSh 86 billion (0.2 percent of GDP). This represents additional income taxes under an agreement with a mining company. 
18. The Government is confident that the careful review that was conducted to identify the above savings will result in successful application of the cash management system and full realization of the savings. The Government will finance the 2011/12 budget deficit without exceeding the PSI ceiling on net domestic financing (NDF) equivalent to 1 percent of GDP. Some further external non-concessional borrowing (ENCB) will be contracted in the coming months, within the PSI limits, to fund infrastructure projects in the 2011/12 budget. Foreign currency financing proposals have been obtained from a number of banks, and a committee has been formed to choose the best options. While the approved 2011/12 budget assumed ENCB of $\$ 822$ million, the corrective measures described above would reduce the ENCB funding need to a projected $\$ 575$ million.

\section{Fiscal policy in 2012/13 and the medium term}

19. The Government is determined to maintain prudent fiscal policy in 2012/13 and beyond. A particular focus will be given to ensuring that recurrent spending is kept to an affordable level. Given Tanzania's development needs, recurrent spending has risen in recent years to fund a growing provision of socially important secondary and tertiary education as well as a broadened range of social programs provided at the local government level (the latter under the "decentralization by devolution" ("D by D") framework). However, as a result, recurrent spending exceeded recurrent incomes (revenues plus grants) in the two most recent years. To ensure that recurrent spending is not debt-financed and to create fiscal space for growth-promoting development spending, the Government intends to ensure that recurrent spending does not exceed 97 percent of recurrent incomes in 2012/13 and 95 percent in 2013/14 and beyond.

20. To achieve this goal, the Government will develop policies in the context of the 2012/13 budget to raise revenue collections as a share of GDP and/or slow the pace of recurrent spending in relation to GDP. The mix of policies to rebalance recurrent incomes and spending will be discussed with the Fund in the context of the fourth PSI review.

21. On the expenditure side, the Government intends to pay particular attention to the scope for expenditure savings in:

- The central government wage bill and associated allowances regime (equivalent to 5.3 percent of GDP in 2011/12);

- $\quad$ The decentralization program for social programs. It will be important not to undermine the delivery of decentralized education, health, water and other programs. At the same time, reforms to strengthen service delivery quality are needed, and the costs of the decentralized expenditure program are high and rising (transfers to local authorities to fund these programs are projected to rise from 5 percent of GDP in 2009/10 to more than 5.6 percent of GDP in 2011/12);

- The student loan program, where annual costs are around 0.6 percent of GDP, and where reforms to strengthen the targeting of assistance are being adopted; and 
- Transfers to public institutions, including colleges, universities, institutes, agencies, boards, and commissions. Excluding the Tanzanian Revenue Authority and the Tanzanian Road Fund, which benefit from important self-financing, current grants to these bodies are projected at 1.6 percent of GDP in 2011/12. Potential savings could be realized by commercializing, privatizing, or closing institutions.

22. In considering the scope for revenue gains, the Government will examine the case for reforms in the following areas:

- Rationalized tax exemptions, including avoidance of preferential treatment under the income and indirect taxes, elimination of tax holidays, and limitation of Special Economic Zone and Export Processing Zone preferences to indirect taxes;

- $\quad$ Inclusion of capital goods and petroleum products in the VAT base, drawing on a planned comprehensive review of Tanzania's VAT regime to be supported by IMF technical assistance in early-2012;

- $\quad$ Increase of user fees and charges to more closely reflect the value of services provided and to compensate for recent inflation; and

- $\quad$ Application of more stringent rules for the transfer of fees collected by MDAs to the consolidated fund.

\section{Public Financial Management}

23. The Government is committed to further strengthening public financial management (PFM) systems so as to achieve a high level of public service delivery. The PFMRP IV program will continue to strengthen Tanzania's PFM systems, focusing on revenue management, planning and budget management, budget execution, transparency and accountability, budget control and oversight, change management, programme monitoring and evaluation. The PFMRP Phase IV will be implemented in a span of five years beginning January 2012.

24. The Government is taking steps to strengthen the internal control systems and develop risk management strategies for central government ministries, departments and agencies (MDAs) and local government authorities (LGAs). The amendment of the Public Finance Act in 2010 established a fully-fledged Internal Audit General Department (IAGD), and in April 2011, an Internal Auditor General was appointed. To ensure a high degree of operational independence, the Head of the IAGD reports directly to the Permanent Secretary (Ministry of Finance), who is also the Paymaster General.

25. In strengthening the management and control of public funds, the Public Finance Act, 2011 (amended 2004) has been reviewed to empower the Paymaster General and the Accountant General to oversee public financial management at the LGA level. Through these changes, the Government has created the post and appointed a new Assistant Accountant General responsible for management of LGA finances. 
26. To better track domestic arrears issues, the Government has begun to compile outstanding liabilities by aging analysis on a quarterly basis since end June 2010 and is providing these data to IMF and other development partners. Currently, the Government has compiled reports for financial year 2010/11 and the first quarter of 2011/12. The Government is determined that the accumulation of domestic arrears in 2010/11 will not be a recurrent issue, and has allocated resources in the 2011/12 budget that are projected to be adequate to clear the arrears stock at end-June 2011. Importantly, the 2011/12 budget for the Ministry of Infrastructures, which accounted for 80 percent of recorded arrears at end-September 2011, is estimated to be adequate to clear these arrears during the course of the year without a carryover into 2012/13.

27. In 2010/11, 13,236 dormant government bank accounts were closed, to promote more efficient supervision and to reduce costs. For the current financial year, 983 dormant accounts have been identified and the closure process is ongoing. Under the new arrangement, each LGA will be limited to six bank accounts.

28. As part of a continuing program to strengthen fiscal transparency, the Ministry of Finance will make available from end-March 2012 on its internet website the full four volumes of public expenditure estimates for the 2011/12 budget as submitted to the National Assembly. In the year ahead, the corresponding 2012/13 budget documents will be added to the Ministry's website.

\section{Debt management}

29. To help ensure the sustainability of Tanzania's national debt, the Government conducted a Debt Sustainability Analysis (DSA) in September 2010 and the report was published in December 2010. The DSA, which incorporated non concessional borrowing, shows that Tanzania's public debt is expected to remain sustainable in the medium and long term. However, the Government recognizes that external debt sustainability is sensitive to the terms of borrowing and effective utilization of funds to finance high priority investment projects. In addition, fiscal debt sustainability is sensitive to large and persistent primary deficits. The Government will continue to control the issuance of guarantees on loans to various public institutions in order to maintain public debt sustainability.

30. The Government is committed to improving its debt monitoring capacity (including through structural benchmarks in the context of the PSI-supported program). The Government has, with the help of IMF-World Bank technical assistance, adopted the Medium Term Debt Strategy (MTDS) tool and published its results in June 2011. The Government has also established a unified single debt database under the Accountant General's Department with the aim of maintaining quality debt data under one roof.

\section{Energy sector policies}

31. Reflecting poor rains in late 2010 and a consequent reduction in hydropower generation capacity, Tanzania has suffered severe power shortages equivalent to as much as 300MW over the past year. In response, the Government has embarked on implementation of 
an Emergency Power Plan (EPP) that has already resulted in a much improved power supply situation. Between July and October 2011, an additional 217.5 MW of capacity was added to the power grid, equivalent to about a third of average demand. This new emergency power generation capacity has, to a large extent, mitigated the power crisis and is expected to be adequate to tide the country through 2012 , after which new generating capacities will come on line.

32. Most of the new generation capacities are based on expensive liquid fuels, rather than low-cost natural gas, whose supplies are currently constrained. The cost to the state power utility, TANESCO, of the additional power purchases considerably exceeds the current average tariff, creating subsidy needs that will diminish when low-cost gas supplies are expanded through a planned new pipeline (see below). The near term additional costs will be met by a combination of tariff adjustment and borrowing by TANESCO, which would allow it to recoup the temporary subsidies over a longer period. Accordingly, TANESCO is in the process of arranging a seven-year domestic currency bank loan amounting to TSh 408 billion (1.0 percent of GDP) which will be repaid out of future tariff revenues. To limit the nearterm subsidy and provide resources for loan repayment, TANESCO has submitted an application for a tariff increase and the Government will work with TANESCO and the energy and water utility regulatory authority, EWURA, to ensure that the regulatory review is completed and that a weighted average tariff adjustment of no less than 40 percent will be implemented by end-December 2011.

33. Going forward, the Government is alert to the fiscal risks that can arise from underfunded state power sector operators, and is determined to ensure that productivity improvements by TANESCO are accompanied by tariff adjustments over the period ahead that ensure its financial viability. The Government will re-examine the adequacy of the tariff structure in the context of the fourth PSI review in light of changes in generation capacity and developments in TANESCO's costs and revenues.

34. The Government is negotiating a $\$ 1.2$ billion financing package (5 percent of GDP) to finance a gas pipeline from Mtwara to Dar Es Salaam for fuel electricity generation and other uses. The planned financing package involves a combination of a concessional loan (75 percent of the total), a commercial loan ( 20 percent), and a contribution by the Government of Tanzania ( 5 percent). The commercial loan will be guaranteed by the government, and will be subject to the ENCB ceiling under the PSI. Given the scale of the project, the Government will ensure that it has a high rate of economic return, and will share with the IMF the technical and economic evaluation of the project.

35. Looking further ahead, Tanzania has recently seen favourable offshore natural gas exploration results. There appear to be good prospects that commercial quantities of natural gas will be confirmed, resulting in multi-billion dollar foreign direct investments in Tanzania's natural gas sector over the next 5 years, and the start of correspondingly large export and budget revenue flows around the end of the current decade. Discussions in Tanzania on how to position the country to best take advantage of this potential resource wealth have been initiated and a Natural Gas Master Plan will be finished by June 2012. In a macroeconomic and budget management context, considerable work will be needed, including design and adoption of a gas/petroleum revenue management bill covering the 
budget treatment of gas revenues; possible launch of a future generations fund to save a portion of the resource wealth; review of Tanzania's tax regime to ensure its adequate cover of the gas sector; and development of staff expertise in the Tanzania Revenue Authority (TRA) on tax issues associated with the development and exploitation of gas. To start the preparation process, the Ministry of Finance, Planning Commission, and TRA will prepare a report by December 2012 identifying what steps will need to be taken to prepare Tanzania's macroeconomic management for the new gas economy, and identifying the nature of any corresponding technical assistance needs. The Government would welcome Fund staff contributions to this advance planning exercise, drawing on best international practice.

\section{Monetary and exchange rate policies}

36. The Bank of Tanzania (BoT) will continue to implement its reserve money targeting framework with a view to keeping core inflation in single digit levels and on track for the medium term goal of 5 percent inflation. The BoT views most of the recent hike in inflation as temporary, and likely to unwind soon on the assumption that good short-rains and harvests will reduce food inflation, and as the improved domestic power supply eases import demand for petroleum to fuel back-up generators, thereby easing pressures on the currency. At the same time, annual growth of average reserve money has been targeted to slowdown to 18 percent in the year ending June 2012, compared to 19 percent recorded in the year ending June 2011. Consistent with this, M3 growth is projected to slow from 23.7 percent in September 2011 to 20.0 percent by June 2012, giving room for credit to private sector to grow by 23 percent. The BoT will monitor risks to inflation closely, and stands ready to further tighten liquidity conditions as needed.

37. In the near term, implementation of the reserve money program will focus on unwinding a surge in reserve money in late-September that led to higher levels of liquidity through October. The BoT is sterilizing this liquidity through a combination of monetary policy instruments. The sterilization effort is also being supported by an increase in the minimum reserve requirements on government deposits from 20 percent to 30 percent effective November 2011 and the decision to raise the BoT's Bank Rate by 200 basis points to 9.58 percent from October 2011 and further to 12.00 percent in November.

38. The exchange rate will remain market determined and the BoT will continue to participate in the foreign exchange market for liquidity management purposes and to smooth out short-term fluctuations in the exchange rate, while maintaining an adequate level of international reserves. The BoT believes that recent currency pressures have been exacerbated by positions taken by banks against the shilling, and accordingly the BoT has reduced the prudential limit on the net open foreign currency position of commercial banks from 20 percent to 10 percent of core capital effective October 26, 2011. In addition, BoT has tightened the enforcement of existing restrictions on non-residents' access to credit facilities and other sources of Tanzania shilling funding, where these are not backed by underlying economic activities. 


\section{Financial sector stability}

39. The BoT continues to enhance financial stability monitoring tools and arrangements in order to ensure stability and soundness of the financial system. A financial regulators forum will be launched shortly to assume joint responsibility for safeguarding the stability of the financial system and to coordinate crisis management and resolution. A draft MOU to establish the Forum has been circulated for comments. With assistance from IMF, the BoT has developed a framework for financial stability monitoring and reporting. The BoT published its first Financial Stability Report in December 2010, followed by a half year review in June 2011. A second Financial Stability Report is scheduled to be published before end-2011. To strengthen the monitoring framework, property price indices and corporate and household balance sheets will need to be developed, and the BoT has requested additional TA from the Fund to address this gap. At the regional level, arrangements for sharing regional financial soundness indicators have been put in place.

40. To promote the soundness and stability of the pension sector, the regulatory structure for the sector was set up in mainland Tanzania in 2010 by the establishment of the Social Security Regulatory Authority (SSRA) under the Social Security (Regulatory Authority) Act, 2008. The SSRA is responsible for the regulation and supervision of the pension sector in mainland Tanzania. Since its inception, the SSRA has taken steps to enhance the stability of the pension sector by: (i) reviewing the Social Security (Regulatory Authority) Act, 2008 in order to create conducive environment for effective regulation and supervision of the sector; (ii) registering the existing social security funds (SSFs) as per section 8 of the Social Security (Regulatory) Authority Act, 2008 in order to introduce regulatory oversight over the sector; and (iii) commissioning an actuarial valuation and portfolio review of all existing SSFs in order to obtain data which will form a basis for various policy decisions regarding the pension sector reform and oversight. Investment guidelines have been developed, and it is expected that the guidelines will be issued before end-March 2012.

41. The insurance sector continues to attract new players, but remains very concentrated, with the largest five insurance companies holding 66 percent of total assets as of March 2011, led by the state-owned NIC Limited with nearly 40 percent of total assets. The company is undergoing a restructuring process prior to considering its privatisation. NIC assets not directly identified with life and non-life business have been sold, with the proceeds used to settle accumulated verified claims and to modernize the business. As a result, injection of government equity into NIC has not been necessary. The level of competition in the insurance sector is expected to grow with the envisaged regulatory changes to allow banks to engage in insurance services.

42. Financial soundness indicators at end-September 2011 indicate that the banking system remains sound, profitable and liquid, in the whole. It was adequately capitalised with the ratios of core capital to total risk weighted assets and other off-balance sheet exposures of 16.8 percent and 17.3 percent, compared with the legal minimum requirements of 10.0 percent and 12.0 percent, respectively. The ratio of non-performing loans (NPLs) declined to 8.1 percent end-September 2011, from 9.3 percent end December 2010. This is partly attributed to efforts taken to improve the quality of loan portfolios by strengthening 
credit underwriting and administration practices, strict follow-up and recovery of NPLs, and a focus of supervisory attention on banking institutions with high NPLs. Overall liquidity remains satisfactory with a ratio of liquid assets to demand liabilities of 41.8 percent, compared to a statutory requirement of 20 percent. The sector also continued to be profitable with return on average assets of 3.0 percent. According to self-assessment on the 25 Basle Core Principles for Effective Banking Supervision conducted in November 2010, the Bank has fully complied with 5 core principles, largely complied with 19 principles but was materially non-compliant with one core principle (i.e. BCP No. 12-Country and Transfer Risks).

43. There have been important developments in the regulation and supervision of banks aimed at enhancing soundness. In the period under review, the risk-based supervision framework was revised to reflect new developments in supervisory principles and standards and incorporate best practices in risk management. Business continuity guidelines have also been issued to banks and financial institutions, and the BoT has initiated the process of developing prudential guidelines for effective supervision of Islamic banking business, the use of which has been growing rapidly. A consultant has been commissioned to spearhead the process of putting in place a comprehensive regulatory and supervisory framework for development finance institutions.

44. Progress has been made in the establishment of a credit reference system. Credit Reference Bureau and Databank Regulations have been finalized and gazetted. A contract for the supply, installation and commissioning of a Credit Reference Databank System at BoT has been signed and the vendor has commenced work. A public notice intended to entice interested and eligible private credit reference bureau operators to apply for a license has also been issued. Potential international credit reference bureau operators have shown interest and some are seeking local partners.

45. The BoT continues to help banks and mobile network operators develop mobile banking through a mobile payment policy partnership. Mobile payment regulations have been drafted by the BoT to promote financial inclusion of the under-banked and unbanked. These will be reviewed by stakeholders by end-December 2011 .

46. In October 2011, the international Financial Action Task Force (FATF) identified Tanzania as one of five jurisdictions not making sufficient progress in improving policies on anti-money laundering and countering the financing of terrorism (AML/CFT). FATF requires that Tanzania take actions to implement significant components of its AML/CFT action plan by February 2012. The Government recognizes the importance for Tanzania's financial sector of a positive FATF assessment and will take determined steps to implement its agreed action plan ahead of the above deadline. In particular, draft legislation to tighten the existing AML framework in line with FATF recommendations and address national jurisdictional issues will be submitted to parliament with a view to approval by end-February. 


\section{Statistical issues}

47. The BoT currently uses the CPI excluding food and energy prices as a proxy for core inflation following revision of the CPI in November 2010. A new core inflation index is being developed, excluding some goods whose prices are subject to volatility due to weather influences and shocks to international prices.

\section{Programme monitoring}

48. Progress in the implementation of the policies under this program will be monitored through assessment criteria (ACs), indicative targets (IT), and structural benchmarks (SBs) as documented in the attached tables. The government requests a modification of the assessment criterion on NIR from measuring NIR on a stock basis to a flow basis to eliminate base effects from performance measurement. The assessment criterion on external debt is requested to be changed to make it cumulative from the beginning of the program instead of the fiscal year to allow for more flexibility in the timing of contracts within the agreed 3-year program ceiling of US $\$ 1.5$ billion. The fourth review is expected to be completed by endJune 2012 and the fifth review by end-December 2012 .

MEFP Table 1. Tanzania: Quantitative Assessment Criteria Under the Policy Support Instrument, June and September 2011

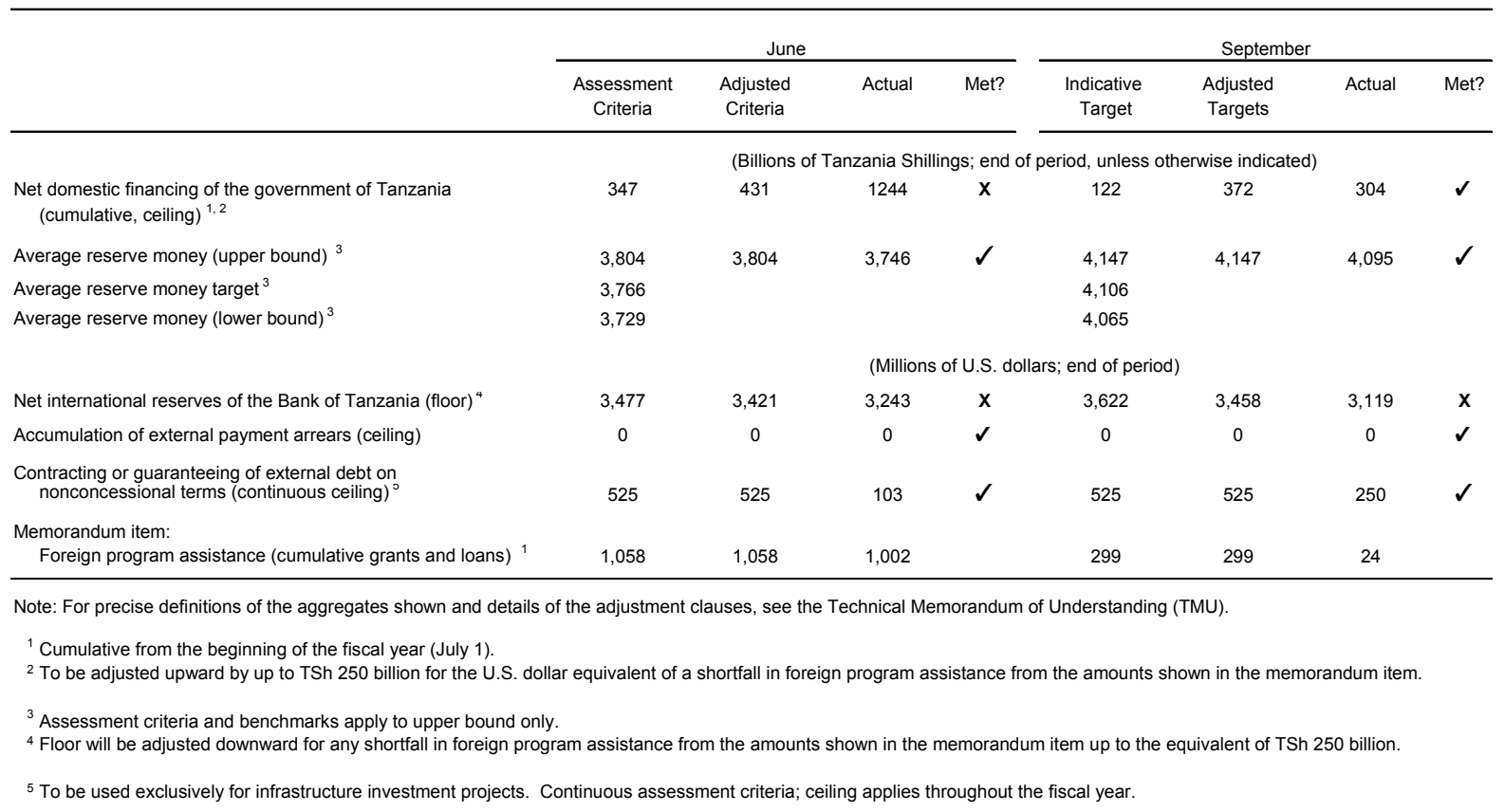


MEFP Table 2. Quantitative Assessment Criteria and Indicative Targets Under the Policy Support Instrument, December 2011-June 2012

\begin{tabular}{|c|c|c|c|c|}
\hline & \multicolumn{2}{|c|}{2011} & \multicolumn{2}{|c|}{2012} \\
\hline & September & December & March & June \\
\hline & Actual & $\begin{array}{l}\text { Assessment } \\
\text { Criteria }\end{array}$ & $\begin{array}{l}\text { Indicative } \\
\text { Targets }\end{array}$ & $\begin{array}{l}\text { Assessment } \\
\text { Criteria }\end{array}$ \\
\hline \multicolumn{5}{|c|}{ (Billions of Tanzania Shillings; end of period, unless otherwise indicated) } \\
\hline $\begin{array}{l}\text { Net domestic financing of the government of Tanzania } \\
\text { (cumulative, ceiling) } \\
1,2\end{array}$ & 304 & 300 & 296 & 397 \\
\hline Average reserve money (upper bound) ${ }^{3}$ & 4,095 & 4,235 & 4,321 & 4,464 \\
\hline Average reserve money target ${ }^{3}$ & & 4,193 & 4,278 & 4,420 \\
\hline Average reserve money (lower bound) ${ }^{3}$ & & 4,151 & 4,235 & 4,376 \\
\hline & \multicolumn{4}{|c|}{ (Millions of U.S. dollars; end of period) } \\
\hline Change in net international reserves of the Bank of Tanzania (floor) ${ }^{4}$ & -124 & 80 & 180 & 258 \\
\hline Accumulation of external payment arrears (continuous ceiling) & 0 & 0 & 0 & 0 \\
\hline $\begin{array}{l}\text { Contracting or guaranteeing of external debt on } \\
\text { nonconcessional terms (continuous ceiling) }\end{array}$ & 350 & 1,050 & 1,050 & 1,050 \\
\hline \multicolumn{5}{|l|}{ Memorandum items: } \\
\hline Foreign program assistance (cumulative grants and loans) ${ }^{1}$ & 24 & 628 & 831 & 1,062 \\
\hline $\begin{array}{l}\text { External nonconcessional borrowing (ENCB) disbursements } \\
\text { to the budget }{ }^{1}\end{array}$ & 191 & 291 & 441 & 575 \\
\hline
\end{tabular}

Note: For precise definitions of the aggregates shown and details of the adjustment clauses, see the Technical Memorandum of Understanding (TMU).

${ }^{1}$ Cumulative from July $1,2011$.

${ }^{2}$ To be adjusted upward by up to TSh 300 billion for the U.S. dollar equivalent of any shortfall in the combined total of foreign program assistance and ENCB from the amounts shown in the memorandum items. To be adjusted downward by any ENCB disbursed for budget financing above the programmed amount for the year as a whole (US\$575 million for 2011/12).

${ }^{3}$ Assessment criteria and benchmarks apply to upper bound only.

${ }^{4}$ Cumulative from July 1, 2011. Floor will be adjusted downward for any shortfall in the combined total of foreign program assistance and ENCB from the amounts shown in the memorandum items up to the equivalent of TSh 300 billion.

5 To be used exclusively for infrastructure investment projects. Continuous assessment criterion; cumulative from July 1, 2010. 
MEFP Table 3. Tanzania: Structural Benchmarks for 2011/12

\section{Measure}

\section{Poverty Reduction Strategy}

Approval by Government of a new National Social Protection Framework (NSPF).

\section{Financial Sector}

Issuance of investment guidelines for pension funds.

\section{Fiscal}

Debt management: Prepare a Medium-Term Debt Strategy consistent with resource needs under the new PRS and reflecting contingent liabilities.

Establish a unified public debt statistics database under a single monitoring unit.

Develop an index for monitoring priority social spending.
Proposed to be dropped. Work on a NSPF has been integrated into an ongoing, broader, national social security reform program.

Proposed delayed to end-March

End-September, 20112012 to complete an actuarial assessment.

End-June, $2011 \quad$ Met.

End-December, 2011 Met.

End-December, 2011 Met. 
MEFP Table 4. New structural benchmarks established in the $3^{\text {rd }}$ PSI review

\begin{tabular}{|c|c|c|c|}
\hline Benchmark & $\begin{array}{l}\text { Macroeconomic } \\
\text { Rationale }\end{array}$ & Date & ๆ \\
\hline $\begin{array}{l}\text { - Regulatory authority approval of an } \\
\text { increase in TANESCO's weighted } \\
\text { average power tariff of not less than } \\
40 \text { percent to reduce its subsidies and } \\
\text { better position it to repay the new loan } \\
\text { that it is contracting to cover operating } \\
\text { costs. }\end{array}$ & $\begin{array}{l}\text { Reduces potential fiscal } \\
\text { costs by strengthening } \\
\text { TANESCO's finances }\end{array}$ & $\begin{array}{l}\text { End- } \\
\text { December } \\
2011\end{array}$ & 32 \\
\hline $\begin{array}{l}\text { - The Ministry of Finance will make } \\
\text { available on a permanent basis on its } \\
\text { internet website copies of the full four } \\
\text { volumes of revenue and public } \\
\text { expenditure estimates as submitted to } \\
\text { and approved by the National Assembly, } \\
\text { starting with the } 2011 / 12 \text { budget. }\end{array}$ & $\begin{array}{l}\text { Improves transparency } \\
\text { of government's } \\
\text { finances and supports } \\
\text { budget monitoring and } \\
\text { control by the public }\end{array}$ & $\begin{array}{c}\text { End-March } \\
2012\end{array}$ & 28 \\
\hline $\begin{array}{l}\text { - The Ministry of Finance will provide to } \\
\text { the IMF the technical and economic } \\
\text { evaluation of the proposed gas pipeline } \\
\text { project. }\end{array}$ & $\begin{array}{l}\text { Promotes evaluation of } \\
\text { large investment } \\
\text { projects by the } \\
\text { government to help } \\
\text { ensures value for } \\
\text { money }\end{array}$ & $\begin{array}{c}\text { End-June } \\
2012\end{array}$ & 33 \\
\hline $\begin{array}{l}\text { - The Ministry of Finance, Planning } \\
\text { Commission, and TRA will prepare a } \\
\text { report identifying steps to be taken to } \\
\text { prepare Tanzania's macroeconomic } \\
\text { management for the new gas economy, } \\
\text { and identifying the nature of any } \\
\text { corresponding technical assistance } \\
\text { needs. }\end{array}$ & $\begin{array}{l}\text { Supports early } \\
\text { preparation for major } \\
\text { macroeconomic } \\
\text { changes in the years } \\
\text { ahead }\end{array}$ & $\begin{array}{l}\text { End- } \\
\text { December } \\
2012\end{array}$ & 34 \\
\hline $\begin{array}{l}\text { - The Government will submit to } \\
\text { parliament draft legislation to tighten the } \\
\text { existing AML framework in line with } \\
\text { FATF recommendations and address } \\
\text { national jurisdictional issues in support of } \\
\text { Tanzania's AML/CFT action plan agreed } \\
\text { with the FATF. }\end{array}$ & $\begin{array}{l}\text { Supports compliance } \\
\text { with international } \\
\text { financial norms and } \\
\text { integrity of financial } \\
\text { system }\end{array}$ & $\begin{array}{l}\text { End- } \\
\text { February } \\
2012\end{array}$ & 45 \\
\hline
\end{tabular}




\section{Attachment II. Technical Memorandum of Understanding on Selected Concepts and Definitions Used in the Monitoring of the PSI-Supported Program}

December 23, 2011

\section{INTRODUCTION}

1. The purpose of this Technical Memorandum of Understanding (TMU) is to describe concepts and definitions that are being used in the monitoring of the quantitative PSI assessment criteria, indicative targets, and structural benchmarks under Tanzania's program supported by the PSI arrangement. The principal data sources are the standardized reporting forms, $1 \mathrm{SR}$ and 2SR, as provided by the Bank of Tanzania to the IMF, and the government debt tables provided by the Accountant General's office.

\section{DEFINITIONS}

\section{Net international reserves}

2. Net international reserves (NIR) of the Bank of Tanzania (BoT) are defined as reserve assets minus reserve liabilities. The change in NIR is calculated as the cumulative change since the beginning of the fiscal year. The BoT's reserve assets, as defined in the IMF BOP manual ( $5^{\text {th }}$ edition) and elaborated in the reserve template of the IMF's special data dissemination standards (SDDS), include: (i) monetary gold; (ii) holdings of SDRs; (iii) the reserve position at the IMF; (iv) all holdings of foreign exchange; and (v) other liquid and marketable assets readily available to the monetary authorities. Reserve assets exclude assets pledged or otherwise encumbered, including but not limited to assets used as collateral or guaranteed for a third party external liability (assets not readily available). The BoT's reserve liabilities include: (i) all short-term foreign exchange liabilities to nonresidents, of original maturities less than one year; and (ii) outstanding purchases and loans from the IMF.

3. NIR are monitored in U.S. dollars, and for program monitoring purposes assets and liabilities in currencies other than U.S. dollars shall be converted into dollar equivalent values using the exchange rates as of June 30, 2011 (as specified below, taken from International Financial Statistics.)

\begin{tabular}{|l|c|}
\hline & $\begin{array}{c}\text { US\$ per } \\
\text { currency unit }\end{array}$ \\
\hline British pound & 1.6008 \\
\hline Euro & 1.4453 \\
\hline Japanese yen & 0.0124 \\
\hline
\end{tabular}




\section{Reserve money and reserve money band}

4. Reserve money is defined as the sum of currency issued by the BoT and the deposits of commercial banks with the BoT. The reserve money targets are the projected daily averages of March, June, September, and December within a symmetrical one percent band. The upper bound of the band serves as the assessment criterion or indicative target.

\section{Net domestic financing of the Government of Tanzania}

5. Net domestic financing of the Government of Tanzania (NDF) includes financing of the budget of the central (union) government of Tanzania ("government") by the banking system (BoT and commercial banks) and the nonbank public. NDF is calculated as the cumulative change since the beginning of the fiscal year in the sum of: (i) loans and advances to the government by the BoT and holdings of government securities and promissory notes (including liquidity paper issued by the BoT for monetary policy purposes), minus all government deposits with the BoT; (ii) all BoT accounts receivable due from the Government of Tanzania that are not included under (i) above; (iii) loans and advances to the government by other depository corporations and holdings of government securities and promissory notes, minus all government deposits held with other depository corporations; and (iv) the outstanding stock of domestic debt held outside depository corporations, excluding: government debt issued for the recapitalization of the NMB and TIB; debt swaps with COBELMO (Russia) and the government of Bulgaria; mortgage on acquired sisal estates; compensation claims; and debt of parastatal companies assumed by the government.

\section{Government deposits at the BoT}

6. Government deposits at the BoT include government deposits as reported in the BoT balance sheet, 1SR -including counterpart deposits in the BoT of liquidity paper issued for monetary policy purposes, and foreign currency-denominated government deposits at the BoT, including the PRBS accounts and the foreign currency deposit account.

\section{External payment arrears}

7. External payment arrears consist of the total amount of external debt service obligations (interest and principal) of the government and the BoT that have not been paid at the time they are due, excluding arrears on external debt service obligations pending the conclusion of debt-rescheduling arrangements. The ceiling on external payment arrears is continuous and applies throughout the year.

\section{Contracting or guaranteeing of external debt on nonconcessional terms}

8. The term "external debt" will have the meaning set forth in Point 9 of the Guidelines on Performance Criteria with Respect to External Debt (Executive Board's Decision No. 6230-(79/140), as amended by Decision No. 14416-(09/91), effective December 1, 2009). External debt will be determined on the basis of currency of denomination of the debt. Government external debt is outstanding debt owed or guaranteed by the Government of Tanzania, the Bank of Tanzania, subnational governments, and companies in which the 
government has at least 50 percent ownership, unless otherwise stipulated. ${ }^{1}$ The ceiling on external debt is continuous and applies throughout the year.

9. Government debt is considered nonconcessional if the grant element is lower than 35 percent, calculated using discount rates based on Organization for Economic Cooperation and Development (OECD) commercial interest reference rates (CIRR), adjusted as appropriate for different maturities. For maturities of less than 15 years, the grant element will be calculated based on 6-month averages of commercial interest rates. For maturities longer than 15 years, the grant element will be calculated based on 10-year averages. This PSI assessment criterion applies not only to debt as defined in Point 9 of the Guidelines on Performance Criteria with Respect to External Debt (Executive Board's Decision No. 6230-(79/140), as amended by Decision No. 14416-(09/91), effective December 1, 2009), but also to commitments contracted or guaranteed for which value has not been received.

\section{Foreign program assistance and program exchange rates}

10. Foreign program assistance is defined as budget support and basket grants and loans received by the Ministry of Finance (MoF) through BoT accounts and accounts at other depository corporations and is calculated as the cumulative sum, since the beginning of the fiscal year, of the receipts from (i) program loans and (ii) program grants. Program assistance does not include nonconcessional external debt as defined in paragraphs 7 and 8.

11. Program exchange rates for the period October 1, 2011 through June 30, 2012 are:

\begin{tabular}{|l|c|c|c|}
\hline & $\begin{array}{c}\text { October- } \\
\text { December 2011 }\end{array}$ & $\begin{array}{c}\text { January-March } \\
2012\end{array}$ & $\begin{array}{c}\text { April-June } \\
2012\end{array}$ \\
\hline $\begin{array}{l}\text { TSh/US\$ averag } \\
\text { e }\end{array}$ & 1,671 & 1,678 & 1,679 \\
\hline $\begin{array}{l}\text { TSh/US\$ } \\
\text { end-period }\end{array}$ & 1,678 & 1,679 & 1,680 \\
\hline
\end{tabular}

\section{AdJUSTERS}

\section{Net international reserves}

12. The end-December 2011, end-March 2012, and end-June 2012 quantitative targets for the change in the BoT's net international reserves will be adjusted downward by the amount in U.S. dollars of any shortfalls in foreign program assistance and external nonconcessional

\footnotetext{
${ }^{1}$ Excluded for these purposes, except if the government offers an explicit guarantee on the debt, are: Tanzania Investment Bank; Tanzania Port Authority; Tanzania Petroleum Development Corporation; Dar es Salaam Rapid Transport Authority; Economic Processing Zones Authority; National Development Corporation; Small Industries Development Organization; National Housing Corporation; National Identity Authority; Dar es Salaam Water and Sewage Authority; and Tanzania Airport Authority.
} 
borrowing (ENCB) financing of the government budget in U.S. dollars (up to a limit of TSh 300 billion). The shortfalls will be calculated relative to projections shown in the Quantitative PSI Assessment Criteria and Indicative Targets Table attached to the applicable Letter of Intent and Memorandum of Economic and Financial Polices of the Government of Tanzania. Each quarterly shortfall will be converted from U.S. dollars to Tanzanian shillings using that quarter's program average exchange rate. The cumulative shortfall will be the sum of all quarterly shortfalls in Tanzanian shillings from the beginning of the fiscal year up to the date of assessment.

\section{Net domestic financing}

13. The end-December 2011, end-March 2012, and end-June 2012 quantitative limits on the net domestic financing of the Government of Tanzania will be adjusted upward for any shortfalls in foreign program assistance and ENCB financing of the government budget in U.S. dollars (up to a limit of TSh 300 billion). The shortfalls will be calculated relative to projections shown in the Quantitative PSI Assessment Criteria and Indicative Targets Table attached to the applicable Letter of Intent and Memorandum of Economic and Financial Polices of the Government of Tanzania. Each quarterly shortfall will be converted from U.S. dollars to Tanzanian shillings using that quarter's program average exchange rate. The cumulative shortfall will be the sum of all quarterly shortfalls in Tanzanian shillings from the beginning of the fiscal year up to the date of assessment.

14. The limits referred to in the previous paragraph will be adjusted downward for any ENCB financing of the government budget in excess of the amount programmed for the year as a whole, indicated in the table referred to in the previous paragraph. 


\section{DATA REPORTING REQUIREMENTS}

15. For purposes of monitoring the program, the Government of Tanzania will provide the data listed in Table 1 below.

Table 1. Summary of Reporting Requirements

\begin{tabular}{|c|c|c|c|}
\hline Information & $\begin{array}{l}\text { Reporting } \\
\text { Institution }\end{array}$ & Frequency & Submission Lag \\
\hline Issuance of government securities. & Bot & Bi-weekly & 1 week \\
\hline Yields on government securities. & Bot & Bi-weekly & 1 week \\
\hline Consumer price index. & NBS & Monthly & 2 weeks \\
\hline $\begin{array}{l}\text { The annual national account statistics in } \\
\text { current and constant prices. }\end{array}$ & NBS & Annually & 6 months \\
\hline $\begin{array}{l}\text { The quarterly national account statistics in } \\
\text { constant prices. }\end{array}$ & NBS & Quarterly & 3 months \\
\hline $\begin{array}{l}\text { Balance sheet of the BoT (1SR) and the } \\
\text { currency composition of foreign assets and } \\
\text { liabilities. }\end{array}$ & Bot & Monthly & 1 week \\
\hline $\begin{array}{l}\text { Consolidated accounts of other depository } \\
\text { corporations and the depository } \\
\text { corporations survey ( } 2 S R \text { and the DCS). }\end{array}$ & Bot & Monthly & 4 weeks \\
\hline $\begin{array}{l}\text { Summary of stock of external debt, } \\
\text { external arrears, and committed } \\
\text { undisbursed loan balances by creditor. }\end{array}$ & Bot & Monthly & 2 weeks \\
\hline External trade developments. & BoT & Monthly & 4 weeks \\
\hline Balance of payments & Bot & Quarterly & 4 weeks \\
\hline $\begin{array}{l}\text { Standard off-site bank supervision } \\
\text { indicators for other depository } \\
\text { corporations. }\end{array}$ & BoT & Quarterly & 6 weeks \\
\hline $\begin{array}{l}\text { Financial Soundness Indicators for other } \\
\text { depository corporations. }\end{array}$ & BoT & Quarterly & 6 weeks \\
\hline $\begin{array}{l}\text { Other depository corporation lending by } \\
\text { activity. }\end{array}$ & Bot & Monthly & 4 weeks \\
\hline Commercial banks interest rate structure. & Bot & Monthly & 4 weeks \\
\hline
\end{tabular}


Table 1. Summary of Reporting Requirements (continued)

\begin{tabular}{|c|c|c|c|}
\hline Information & $\begin{array}{l}\text { Reporting } \\
\text { Institution }\end{array}$ & Frequency & Submission Lag \\
\hline $\begin{array}{l}\text { Summary table of: (i) average reserve } \\
\text { money; (ii) net domestic financing of the } \\
\text { government; (iii) stock of external arrears; } \\
\text { (iv) new contracting or guaranteeing of } \\
\text { external debt on nonconcessional terms; } \\
\text { and (v) net international reserves. }{ }^{1}\end{array}$ & BoT and MoF & Quarterly & 4 weeks \\
\hline $\begin{array}{l}\text { Amount of payment claims outstanding of } \\
\text { more than } 30 \text { days, } 60 \text { days, and } 90 \text { days } \\
\text { for all government ministries. For each } \\
\text { ministry, total claims outstanding to be } \\
\text { divided into: (i) unverified claims; and } \\
\text { (ii) claims verified but not yet paid. }\end{array}$ & $\mathrm{MoF}$ & Quarterly & 4 weeks \\
\hline $\begin{array}{l}\text { The flash report on revenues and } \\
\text { expenditures. }\end{array}$ & MoF & Monthly & 4 weeks \\
\hline The TRA revenue report & TRA & Monthly & 4 weeks \\
\hline The monthly domestic debt report. ${ }^{1}$ & $\mathrm{MoF}$ & Monthly & 4 weeks \\
\hline $\begin{array}{l}\text { Monthly report on central government } \\
\text { operations. }\end{array}$ & MoF & Monthly & 4 weeks \\
\hline $\begin{array}{l}\text { Detailed central government account of } \\
\text { disbursed budget support grants and } \\
\text { loans, and external debt service due and } \\
\text { paid. }\end{array}$ & $\mathrm{MoF}$ & Monthly & 4 weeks \\
\hline $\begin{array}{l}\text { Detailed central government account of } \\
\text { disbursed donor project support grants and } \\
\text { loans. }\end{array}$ & MoF & Monthly & 4 weeks \\
\hline $\begin{array}{l}\text { Statement on new external loans } \\
\text { contracted and guarantees provided by the } \\
\text { entities listed in paragraph } 7 \text { of the TMU } \\
\text { during the period including terms and } \\
\text { conditions according to loan agreements. }\end{array}$ & MoF & Quarterly & 4 weeks \\
\hline
\end{tabular}

\footnotetext{
${ }^{1}$ The MoF and BoT will reconcile data on BoT claims on the government, to ensure that such claims recorded in the BoT balance sheet are the same as those reported by the Accountant General of the MoF.
} 


\title{
INTERNATIONAL MONETARY FUND \\ UNITED REPUBLIC OF TANZANIA
}

\section{Third Review Under the Policy Support Instrument and Request for Waivers and Modification of Assessment Criteria-Informational Annex}

\author{
Prepared by the African Department \\ (In consultation with other departments)
}

January 11,2012

\section{- $\quad$ Relations with the Fund.}

- Joint Bank-Fund Work Program. Describes Bank-Fund collaboration during 2012.

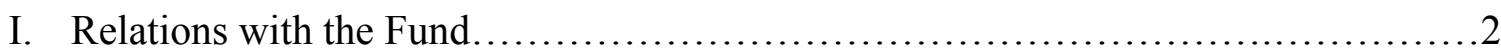

II. Joint Bank-Fund Work Program............................................ 5 


\section{TANZANiA-Relations WITH THE FUND}

(As of December 31, 2011)

I. Membership Status: Joined on September 10, 1962; Article VIII

II. General Resources Account

Quota

Fund holdings of currency

Reserve Tranche Position

III. SDR Department

Net cumulative allocation

Holdings

IV. Outstanding Purchases and Loans

ESF Arrangements

ECF Arrangements
SDR million

198.90

188.90

10.00

SDR million

190.51

156.79

SDR million

218.79

9.52
Percent of quota 100.00 94.97 5.03

Percent of allocation

100.00

82.30

Percent of quota

110.00

4.79

\section{Latest Financial Arrangements}

$\begin{array}{lcccc}\text { Type } & \begin{array}{c}\text { Date of } \\ \text { arrangement }\end{array} & \begin{array}{c}\text { Expiration } \\ \text { date }\end{array} & \begin{array}{c}\text { Amount approved } \\ \text { (SDR million) }\end{array} & \begin{array}{c}\text { Amount drawn } \\ \text { (SDR million) }\end{array} \\ & \text { May 29, 2009 } & \text { June 14, 2010 } & 218.79 & 218.79 \\ \text { ECF }^{1} & \text { Aug 16, 2003 } & \text { Feb 26, 2007 } & 19.60 & 19.60 \\ \text { ECF }^{1} & \text { Apr 04, 2000 } & \text { Aug 15, 2003 } & 135.00 & 135.00\end{array}$

${ }^{1}$ Formerly PRGF.

VI. Projected Payments to Fund (SDR million; based on existing use of resources and present holdings of SDRs)

Principal

$\frac{2012}{1.96} \quad \frac{2013}{2.24}$

$\underline{0.04}$

$\underline{2.00}$

$\begin{array}{r}2014 \\ 18.15 \\ \underline{0.59} \\ 18.75 \\ \hline\end{array}$

$\underline{18.75}$

\begin{tabular}{rrr}
$\frac{2015}{43.73}$ & $\underline{2016}$ \\
$\underline{0.52}$ & $\underline{0.41}$ \\
$\underline{44.25}$ & $\underline{45.01}$ \\
\hline
\end{tabular}




\title{
VII. Implementation of HIPC Initiative
}

Enhanced

I. Commitment of HIPC assistance

Framework

Decision point date

April 2000

Assistance committed

by all creditors (US $\$$ million) ${ }^{1}$

Of which: IMF assistance (US\$ million)

(SDR equivalent in millions)

Completion point date

November 2001

II. Disbursement of IMF assistance (SDR million)

Assistance disbursed to the member

Interim assistance

Completion point balance

Additional disbursement of interest income ${ }^{2}$

Total disbursements

${ }^{1}$ Assistance committed under the original framework is expressed in net present value (NPV) terms at the completion point, and assistance committed under the enhanced framework is expressed in NPV terms at the decision point. Hence these two amounts can not be added.

${ }^{2}$ Under the enhanced framework, an additional disbursement is made at the completion point corresponding to interest income earned on the amount committed at the decision point but not disbursed during the interim period.

\section{Implementation of Multilateral Debt Relief Initiative (MDRI)}

I. MDRI-eligible debt (SDR million) ${ }^{1}$

Financed by: MDRI Trust

207.00

Remaining HIPC resources

II. Debt Relief by Facility (SDR million)

\section{Eligible Debt}

\author{
$\underline{\text { Delivery }}$ \\ Date \\ $\underline{\text { GRA }}$ \\ $\underline{\text { PRGF }}$ \\ $\underline{\text { Total }}$ \\ January 2006 \\ N/A \\ 234.03 \\ 234.03
}

\footnotetext{
${ }^{1}$ The MDRI provides 100 percent debt relief to eligible member countries that qualified for the assistance. Grant assistance from the MDRI Trust and HIPC resources provide debt relief to cover the full stock of debt owed to the Fund as of end-2004 that remains outstanding at the time the member qualifies for such debt relief.
} 


\section{Safeguards Assessments}

Safeguards assessments of the Bank of Tanzania (BoT) were completed in December 2003, August 2008, and November 2009. The 2009 assessment found that, while the BoT had taken steps to strengthen its safeguards framework, risks remained due to delays in implementing certain priority recommendations from the 2008 voluntary assessment. The external audit and financial reporting continued to comply with international standards, but the audited results were published with significant delays. The assessment recommended a bank-wide risk assessment, outlined steps needed to modernize the internal audit function, and proposed measures to enhance transparency of financial, control and compliance matters. The BoT has since taken steps to improve the timeliness of its financial audits and the financial statements for 2009/10 were published within the statutory deadline. The implementation of other measures is in progress.

\section{Exchange Rate Arrangement}

The currency of Tanzania is the Tanzania shilling. Under the revised classification methodology, Tanzania has a floating exchange rate arrangement. The official exchange rate is determined in relation to the rate established in the interbank market for foreign exchange. The weighted average rate in the interbank market was TSh 1,582.48 per U.S. dollar as of December 29, 2011. The exchange system is free of restrictions on the making of payments and transfers for current international transactions.

\section{Article IV Consultation}

The most recent Article IV consultation was concluded on May 6, 2011 (Country Report No. 11/105).

\section{Resident Representative}

Mr. John Wakeman-Linn has been the Senior Resident Representative since August 2010. 
VI. TAnZania-JoInt BANK-Fund Work Program, 2012

\begin{tabular}{|c|c|c|c|}
\hline Title & Products & $\begin{array}{l}\text { Provisional } \\
\text { timing of } \\
\text { missions } \\
\text { (if relevant) }\end{array}$ & $\begin{array}{l}\text { Expected delivery } \\
\text { date }\end{array}$ \\
\hline \multicolumn{4}{|c|}{ A. Mutual information on relevant work programs } \\
\hline \multirow[t]{4}{*}{$\begin{array}{l}\text { Key elements of } \\
\text { World Bank work } \\
\text { program in next } \\
12 \text { months }\end{array}$} & $\begin{array}{l}\text { 1. The work program will continue to } \\
\text { concentrate on areas within the existing and } \\
\text { new lending portfolio: primary, secondary, } \\
\text { and higher education; health sector } \\
\text { development; water and sanitation; social } \\
\text { action fund; agriculture; agribusiness } \\
\text { development (Southern Agricultural Growth } \\
\text { Corridor); natural resource management } \\
\text { including minerals; environment; road and } \\
\text { railway infrastructure; power sector } \\
\text { restructuring; housing finance; statistical } \\
\text { capacity; urban infrastructure; fiscal } \\
\text { decentralization; private sector } \\
\text { competitiveness; and public sector reform. } \\
\text { Increasing regional portfolio in agriculture, } \\
\text { health, finance, power, and environment. }\end{array}$ & & Continuous \\
\hline & $\begin{array}{l}\text { 2. Public expenditure review with the focus } \\
\text { on rapid budget analysis (RBA); Public } \\
\text { Investment Management (PIM) Assessment } \\
\text { focusing on the energy sector; and Public } \\
\text { Expenditure Review (PER) with new content } \\
\text { on Value for Money (VFM) in the health } \\
\text { sector. }\end{array}$ & & $\begin{array}{l}\text { April } 2012 \text { (PIM } \\
\text { Assessment in the } \\
\text { energy sector); } \\
\text { April } 2012 \text { (VFM } \\
\text { in the health } \\
\text { sector); and } \\
\text { November } 2012 \\
\text { (RBA) }\end{array}$ \\
\hline & $\begin{array}{l}\text { 3. Debt management reform plan mission by } \\
\text { PREM (Economic Policy and Debt } \\
\text { Department) will take place, focusing on } \\
\text { institutional fragmentation and operational } \\
\text { risk management issues. }\end{array}$ & $\begin{array}{l}\text { Mission (from } \\
\text { late January or } \\
\text { early February) }\end{array}$ & February 2012 \\
\hline & $\begin{array}{l}\text { 4. Ninth Poverty Reduction Support Credit } \\
\text { (PRSC-9), the first operation of the new series } \\
\text { of three annual programmatic development } \\
\text { policy operations (DPOs) to contribute to } \\
\text { general budget support (GBS) in Tanzania, is } \\
\text { being prepared for Board approval in March }\end{array}$ & $\begin{array}{l}\text { Regional } \\
\text { Operations } \\
\text { Committees } \\
\text { meeting } \\
\text { (January 19, } \\
\text { 2012); Appraisal }\end{array}$ & March 2012 \\
\hline
\end{tabular}




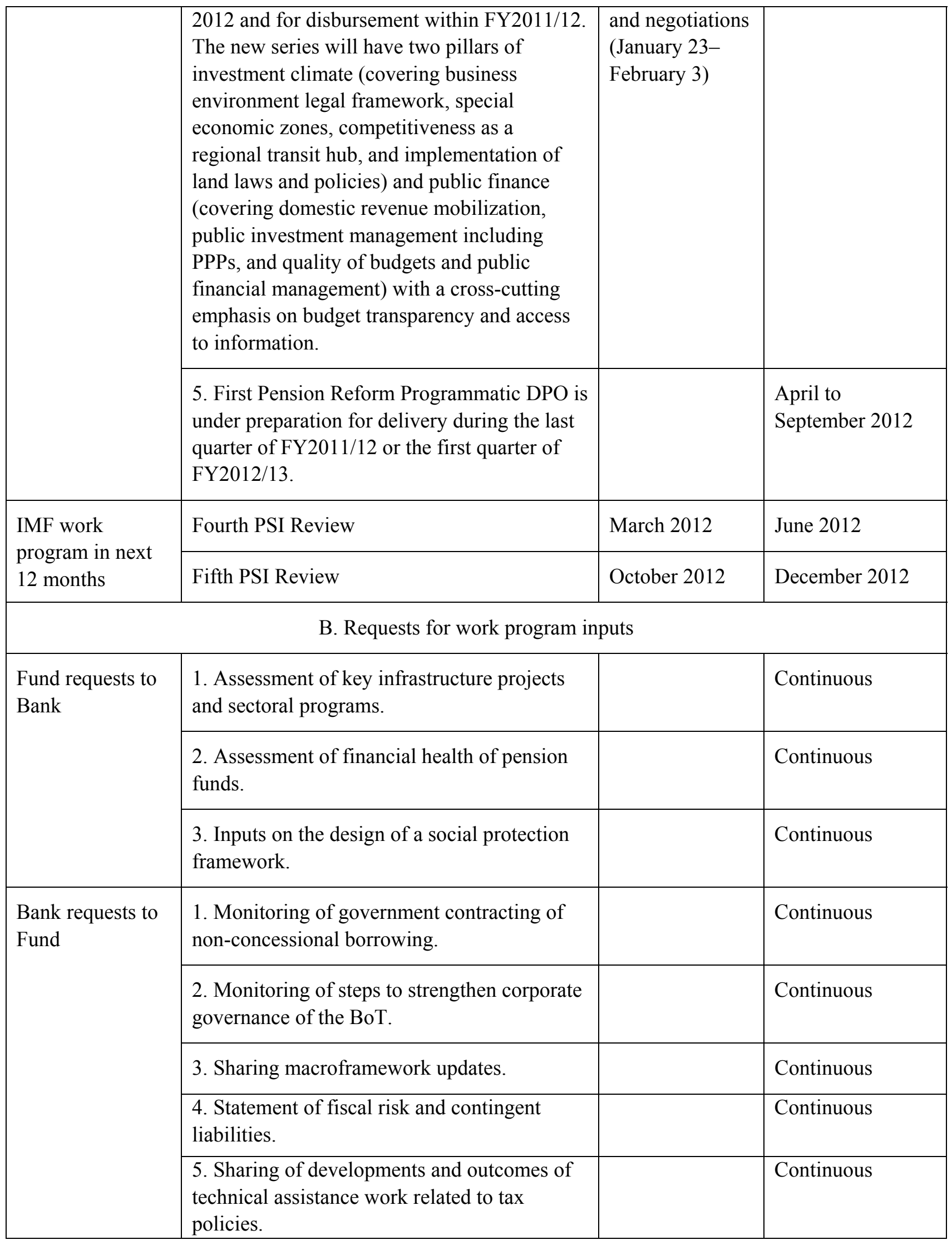




\begin{tabular}{|l|l|l|l|}
\hline \multicolumn{3}{|c|}{ C. Agreement on joint products and missions } \\
\hline \multirow{2}{*}{$\begin{array}{l}\text { Joint products in } \\
\text { next 12 months }\end{array}$} & 1. Collaborate on a joint DSA & March 2012 & April 2012 \\
\cline { 2 - 4 } & $\begin{array}{l}\text { 2. Collaborate together with other } \\
\text { development partners on strengthening the } \\
\text { Public Expenditure Review (PER) dialogue } \\
\text { process. }\end{array}$ & Continuous \\
\cline { 2 - 4 } & $\begin{array}{l}\text { 3. Collaborate on public financial } \\
\text { management reform program in the context of } \\
\text { multi-donor support of the Public Financial } \\
\text { Management Reform Program and/or } \\
\text { GBS/PRSC. }\end{array}$ & Continuous \\
\hline
\end{tabular}


Press Release No. 12/17

FOR IMMEDIATE RELEASE

January 20, 2012
International Monetary Fund

Washington, D.C. 20431 USA

\section{IMF Executive Board Completes Third Review Under Policy Support Instrument for Tanzania}

The Executive Board of the International Monetary Fund (IMF) today completed the third review under the Policy Support Instrument (PSI) for Tanzania. In completing the review, the Board approved waivers of the nonobservance of end-June 2011 assessment criteria on net domestic financing and on net international reserves. The Board also approved a modification of assessment criteria for end-December 2011 for net international reserves and external debt.

The PSI for Tanzania was approved on June 4, 2010 (see Press Release No. 10/227).). The IMF's framework for PSIs is designed for low-income countries that may not need, or want, IMF financial assistance, but still seek IMF advice, monitoring and endorsement of their policies. PSIs are voluntary and demand driven (see Public Information Notice No. 05/145).

Following the Executive Board's discussion on Tanzania, Mr. Naoyuki Shinohara, Deputy Managing Director and Acting Chair, stated:

"Tanzania has weathered the global financial crisis well, and economic growth remains robust. Nevertheless, downside risks remain, and inflation and fiscal deficits have increased.

"To help rebuild macroeconomic buffers, the authorities have decided to tighten the fiscal stance for 2011/12 by eliminating non-priority recurrent spending and delaying some development spending. Further fiscal adjustment will be needed in 2012/13 to strengthen debt sustainability while creating space for infrastructure investment and social spending. The authorities have also raised electricity tariffs to help pay for emergency near-term generating costs and strengthen power utility finances for the medium term. 
"The authorities have tightened monetary conditions to contain inflation. They will need to remain vigilant, and tighten domestic liquidity further if inflationary pressures were to rise. International reserves should be maintained at an adequate level to provide a buffer against external shocks.

“Tanzania's prospects of becoming a major producer of natural gas by the end of the decade appear good. There could be large foreign direct investment inflows over the next five years, and a substantial increase in exports and government revenue beginning around 2020. Successfully managing this future gas wealth will be critical. The authorities are appropriately planning to review over the coming year the macroeconomic policies and institutions that are needed to meet this challenge", Mr. Shinohara added. 


\section{Statement by Mr. Moeketsi Majoro, Executive Director for the United Republic of Tanzania}

January 20, 2012

1. My Tanzanian authorities remain determined to persevere with prudent and sound macroeconomic policies in spite of the challenges emanating from the second round of heightened global economic uncertainties coupled with repetitive drought-related demand and supply shocks for food in the greater East African region. They remain committed to deepen further their structural reform agenda and strengthen the country's macroeconomic fundamentals and policy framework to further shore up the economy's broad-based and inclusive growth. With their policy stance and unrelenting support from the international community and policy guidance from the Fund, my authorities are confident that they stand a good chance of mitigating the strong headwinds emanating from the current round of commodity price surges and the fallout from the heightening global economic uncertainty.

2. My authorities are appreciative of the Fund's constructive engagement and support under the PSI. They thank staff for the candid policy dialogue and advice under the program and for the related technical assistance packages. Going forward, the authorities are determined to further strengthen Tanzania's macroeconomic fundamentals and policies, persevere with the reform effort including laying a good foundation for the management of the new gas economy, strengthen their engagement with the Fund and their development partners, and achieve key national and regional objectives set out in their respective policy guidelines and protocols.

3. To this end, and in view of the strong performance under the program - with all but two end-June 2011 quantitative performance/assessment criteria observed with good margins, and implementation of structural reforms on track - they request Directors' support for the completion of the third review of the PSI. With corrective actions in place, they also request the waivers for the nonobservance of end-June assessment criteria on net domestic financing and on net international reserves; and Directors' support for the modification of the end-December 2011 assessment criteria for net international reserves and external debt.

\section{Recent economic developments and respective policy environment}

4. Tanzania's strengthened economic fundamentals have helped the economy maintain a strong growth momentum despite the successive rounds of global economic downturns. That notwithstanding, the heightened global economic uncertainties and region-specific shocks have generated new sets of challenges that may further dampen economic activity. Consequently, real GDP that had recovered from 6.0 percent in 2009 to 7.4 percent in 2010 is now projected to ease to 6.0 percent in 2011, mainly due to the deterioration in the external environment coupled with weather-induced power shortages. However, prospects for medium term growth remain positive. The coming on stream of government's investments in additional power generation including the new gas pipeline coupled with stronger investment in key economic and social sectors will augur well for economic growth in the medium term. In addition, the coming on stream of the new gas extraction, improvement of the external 
economic environment and normalization of the weather conditions are also expected to support and raise Tanzania's economic growth trajectory in the medium term.

5. The protracted high global food and fuel prices, the repetitive regional drought conditions, an increase in domestic power tariffs and the recent exchange rate volatility continue to exert pressure on domestic inflation. Reemergence of inflationary pressures has raised new concerns and, in this regard, the Bank of Tanzania (BoT) has adopted a tightened monetary policy stance to forestall the inflationary challenges. Additionally, the BoT is strengthening the operation of its policy instruments for sterilization of liquidity and broadening of its monetary policy transparency. The authorities remain committed to maintaining the flexible exchange rate regime which they reaffirm has served the country well and has boosted export diversification.

6. The external balances weakened slightly in 2010/2011 mainly due to a sharp rise in the Import costs (mainly fuel) while exports recorded a double digit growth reflecting strong performance in traditional and manufactured exports and favorable commodity prices, especially of gold. Official gross reserves by end-September 2011 are estimated to decline to USD 3.5 billion equivalent to about 3.7 months of import of goods and services (down from 4.4 months of import cover at end-June 2011). This was also due to delays in donor disbursements and non-concessional borrowing, as well as negative valuation effects on reserves held in euros and sterling when these currencies depreciated against the dollar.

7. My authorities' fiscal policy in FY 2010/2011 broadly remained within the PSI targets, mainly due to a proactive management of spending and significant improvement in revenue performance. The authorities' cash-budgeting system helped contain monthly expenditure within available resources and meet the domestic financing target. The overall deficit of 6.9 percent of GDP was marginally above the program goal of 6.5 percent of GDP. On the revenue front, the 2010/2011 performance was well above program target by 0.4 percent of GDP reflecting the authorities' perseverance with their enhanced revenue collection efforts and related reforms.

8. Reflecting the growing development financing needs especially with respect to education, health and infrastructure, the 2011/12 budget was approved to support the authorities' efforts to meet these needs. However, with downside risks emanating from uncertainties in external financing and rising inflationary pressures, my authorities, through a broad-based expenditure savings and strict application of cash management coupled with enhanced revenue collection efforts, have revised downwards the projected fiscal deficit to 6.6 percent of GDP. They are, however, keenly mindful of the need to sustain spending on social sectors and infrastructure consistent with their broad-based and inclusive growth objectives. Data as of end-December 2011 further indicates continued improvement in domestic revenue collection and the full year performance is projected to exceed the program target. The authorities are confident that the downside risks emanating from the impact of the protracted power shortages on corporate activity and profits on the level of revenue collections will in the near term abate as new power generation capacities come on line. The recent electricity tariff adjustment will also enhance revenue for the power utility and thereby reduce potential subsidies. They are aware of the difficulties entailed in modifying the mining sector tax 
regime in light of the existing agreements, nonetheless they remain focused on broadening the tax base, and strengthening customs and tax administration. They view the continued Fund TA support in tax administration as valuable inputs into their reform effort.

9. The government has made progress in raising funds from external non-concessional borrowing strictly within the PSI targets. Negotiations are in progress with various banks and institutions for an amount of up to US\$575 million for the current financial year as per the program targets. The authorities are confident that they will make use of the entire nonconcessional borrowing window for this financial year.

10. The banking system remains sound and continues to weather the impact of the global economic headwinds well, supported by a strong capital base. The upturn in the level of nonperforming loans (NPLs) has reversed. Nevertheless, the authorities continue to monitor these aggregates carefully. The authorities are aware that the key challenge facing Tanzania's financial sector today is to ensure that the pension funds are strong, and managed professionally, in the interests of the existing and prospective pensioners. In that regard, they have attached high priority to strengthen the operations of the new regulator, the Social Security Regulation Authority, with, inter alia, publication of the investment guidelines for the social security funds.

\section{Medium-term policy framework and reforms}

\section{Fiscal policy and related reforms}

11. My authorities' fiscal policy, going forward, will strive to strike and maintain an appropriate balance between the objective of stimulating economic growth and maintaining macroeconomic stability. To achieve these objectives, the authorities intend to implement further measures to reduce the overall fiscal deficit through expenditure restraint and revenue enhancing measures. On the expenditure restraint, the authorities intend to contain recurrent spending especially on the wage bill and create the fiscal space necessary for sustained budgetary spending levels for priority services, and modest scaling-up of investment in infrastructure and primary sectors to shore-up economic growth. Deepening of structural reforms to improve budget execution and efficiency will continue to be prioritized.

12. To maintain spending in these priority areas while embarking on a fiscal consolidation path, the authorities will continue to strengthen domestic and concessional resource mobilization in the medium term. The authorities have embarked on further revenue enhancing reforms with the view of raising the revenue yield. Among the key revenue reform measures, the scope of tax expenditures will be streamlined substantially and tax holidays eliminated. The VAT base will be enlarged drawing on the planned comprehensive review of the VAT regime with the support of the Fund TA. Internal control systems will be strengthened further and risk management strategies developed for all spending agencies. Cognizant of the overall resource limitations, the authorities intend to facilitate greater inflows of FDI and private sector participation through PPPs, and fully utilize this year's nonconcessional financing window of US\$575 million. They will especially remain vigilant of the contingent liabilities arising from greater use of FDI and PPPs, and debt sustainability implications of non-concessional funding. To that end, they are building capacities in these 
areas, and with the help of the Fund and the World Bank they have adopted the Medium Term Debt Strategy (MTDS) tool and published its results in June 2011.

\section{Monetary and exchange rate policies}

13. My authorities will continue with the tight monetary policy stance with the objective to bring down inflation to the BoT's policy target of 5 percent in the medium term. The authorities will remain vigilant and closely monitor risks to inflation, especially those arising from the protracted high global commodity prices and regional supply shocks. To achieve the objective of anchoring its monetary policy on low and stable inflation, while supporting growth through maintaining sufficient liquidity in the financial system, the BoT will set its policy rate consistent with its disinflationary path. The BoT will further bolster its monetary policy instruments, rely substantially on foreign exchange sales for sterilization of liquidity, and continue to improve liquidity forecasting. The BoT is committed to improve the transparency of its policy stance and promote orderly financial markets.

14. On the exchange rate, the BoT reaffirms its commitment to a market determined exchange rate, and continue to see this as the first line of defense against external shocks. In this regard, the BoT will continue to participate in the foreign exchange market for liquidity management purposes and to smooth out short-term fluctuations in the exchange rate. Additionally, the BoT will enforce the existing restrictions on non-residents' access to credit facilities and other sources of the Tanzania shilling funding, where these are not backed by underlying economic activities. The authorities will also seek to make progress in the preparation for and negotiations of the East African Monetary Union. To that end, they and other EAC partners continue to appreciate the Fund's support in key areas of the preparations and, going forward, welcome further structural support.

\section{Financial sector stability}

15. Financial soundness indicators at end-September 2011 indicate that the banking system remains sound, profitable and liquid, and adequately capitalized, and NPLs have declined reflecting improved quality of loan portfolios. The BoT will continue to strengthen its financial stability monitoring tools and arrangements to ensure stability and soundness of the financial system. To that end, a financial regulators' forum will be launched shortly to assume joint responsibility for safeguarding the stability of the financial system and to coordinate crisis management and resolution. Thanks to the Fund's support, the BoT's framework for financial monitoring and reporting has taken root with the second Financial Stability Report to be published during the first quarter of 2012. Following the full operationalization of the Social Security Regulatory Authority for the pension sector, the investment guidelines prepared by the BoT will be issued in the near term.

\section{Energy policies and the new gas economy}

16. At the time of the second review the authorities indicated their intention to commission, on an emergency basis, additional power generation capacity to meet the acute shortage of electricity in the tune of $300 \mathrm{MW}$. They have since implemented their Emergency Power Plan (EPP) that has added an additional 217.5 MW to the power grid. Though this 
new capacity has considerably mitigated the power crisis, it is based on expensive hydrocarbons the supply of which is constrained but also has significant implications on the import bill and the government budget. In this regard, the authorities have embarked on a system of periodic tariff adjustment and measures to enhance productivity improvements in power generation. On the later, the authorities intend to commission shortly the construction of a $520 \mathrm{~km}$ gas pipeline, the financing of which is well within the PSI targets, to supply natural gas for power generation and other uses. It is envisaged that the new sources of energy will substantially improve the supply of electricity and other sources of energy for industrial, services and household use at a much lower cost than current.

17. The authorities have recorded substantive natural gas exploration results. When fully confirmed the new natural gas economy is envisaged to emerge with a potential multi-billion dollar investments over the medium term with a correspondingly large export and budget revenue flows. To best take advantage of this potential natural resource wealth, the authorities are embarking on the preparation of a Natural Gas Master Plan to be finalized later this year. They are aware that in a macroeconomic and budget management context, considerable work will be needed also to position the country to manage the new natural gas economy as well as mitigate the related Dutch disease effects. They, in this regard, request Fund's comprehensive TA support under the Topical Trust Fund for Managing Natural Resources Wealth (MNRW-TTF).

\section{Conclusion}

18. The authorities' macroeconomic fundamentals and continued commitment to strong and sound policies helped the economy to sustain a growth momentum despite the heightened global economic uncertainties. They are aware of the build-up of new risks and are, therefore, prepared to take appropriate measures to protect the gains in the country's macroeconomic stability. In the near term, and notwithstanding the need for fiscal consolidation, the authorities are determined to ensure that the program focuses on sustaining macroeconomic stability, enhancing domestic resource mobilization, promoting broad-based and pro-poor growth, and creating fiscal space for increased investment in infrastructure, primary sectors and core MDG clusters. They are starting to focus on the future opportunities and challenges arising from the new natural gas economy. Going forward, they request continued support from the Fund, other international financial institutions and development partners in their efforts to strengthen macroeconomic stability, exploit new growth drivers, and achieve their development goals. 